\title{
Modeling He-rich subdwarfs through the hot-flasher scenario
}

\author{
M. M. Miller Bertolami ${ }^{1,2, \star}$, L. G. Althaus ${ }^{1,2, \star \star}$, K. Unglaub $^{3}$, and A. Weiss ${ }^{4}$ \\ 1 Facultad de Ciencias Astronómicas y Geofísicas, Universidad Nacional de La Plata, Paseo del Bosque s/n, (1900) La Plata, \\ Argentina \\ e-mail: mmiller@fcaglp.unlp.edu.ar \\ 2 Instituto de Astrofísica La Plata, UNLP-CONICET, Argentina \\ 3 Dr. Remeis-Stenwarte Bamberg, Sterwartstr. 7, 96049 Bamberg, Germany \\ ${ }^{4}$ Max-Planck-Institut für Astrophysik, Karl-Schwarzschild-Str. 1, 85748, Garching, Germany
}

Received 11 June 2008 / Accepted 22 August 2008

\begin{abstract}
We present 1D numerical simulations aimed at studying the hot-flasher scenario for the formation of He-rich subdwarf stars. Sequences were calculated for a wide range of metallicities and physical assumptions, such as the stellar mass at the moment of the helium core flash. This allows us to study the two previously proposed flavors of the hot-flasher scenario ("deep" and "shallow" mixing cases) and to identify a third transition type. Our sequences are calculated by solving simultaneously the mixing and burning equations within a diffusive convection picture, and in the context of standard mixing length theory. We are able to follow chemical evolution during deep-mixing events in which hydrogen is burned violently, and therefore able to present a homogeneous set of abundances for different metallicities and varieties of hot-flashers. We extend the scope of our work by analyzing the effects of non-standard assumptions, such as the effect of chemical gradients, extra-mixing at convective boundaries, possible reduction in convective velocities, or the interplay between difussion and mass loss. Particular emphasis is placed on the predicted surface properties of the models.

We find that the hot-flasher scenario is a viable explanation for the formation and surface properties of He-sdO stars. Our results also show that, during the early He-core burning stage, element diffusion may produce the transformation of (post hot-flasher) He-rich atmospheres into He-deficient ones. If this is so, then we find that He-sdO stars should be the progenitors of some of the hottest sdB stars.
\end{abstract}

Key words. stars: evolution - stars: horizontal-branch - stars: subdwarfs - stars: mass-loss

\section{Introduction}

Hot subluminous stars are an important population of faint blue stars that can roughly be grouped into the cooler sdB stars, whose spectra display no or only weak helium lines, and the hotter sdO stars, which have higher helium abundances and can even be dominated by helium. Subluminous O, B stars were identified with stars populating the hot end of the horizontal branch (HB) of some globular and open clusters (de Boer et al. 1997). While the sdB stars form a homogeneous spectroscopic class, a large variety of spectra is observed among sdO stars (Lemke et al. 1997). This diversity of spectra and the heliumenhanced surface abundances observed in many of these stars pose a challenge to our understanding of stellar evolution. As a consequence, some non-canonical evolutionary scenarios were proposed for their formation. Among them, the merger of two white dwarfs (Saio \& Jeffery 2000) and a core helium flash after departure from the red giant branch (i.e. "hot-flasher scenario") offer the most promising explanations of their formation (Ströer et al. 2007; Moehler et al. 2007; Hirsch et al. 2008). The second possibility is the subject of the present article. The hot-flasher scenario was proposed to explain the existence and characteristics of blue hook stars (such as helium or carbon enhancement) of some globular clusters e.g. wCen and NGC 2808

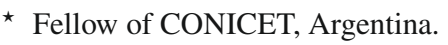

$\star \star$ Member of the Carrera del Investigador Científico y Tecnológico, CONICET, Argentina.
}

(D'Cruz et al. 2000; Moehler et al. 2002; Moehler et al. 2004; Moehler et al. 2007). The coexistence of extreme horizontal branch (EHB) stars and helium core white dwarfs in globular clusters (Calamida et al. 2008) also agrees with a natural prediction of the hot-flasher scenario (see Castellani et al. 2006). Although in this scenario mass loss must be increased artificially, from values usually adopted in the modeling of low mass AGB stars, the driving mechanism behind RGB mass loss is not well understood (Espey \& Crowley 2008). Differences in metallicity, initial He abundances or rotational velocities may, in principle, produce enhanced mass loss rates (the last two by an increase in the RGB-tip luminosity). The later is particularly interesting in view of the fact that some $\mathrm{He}-\mathrm{sdO}$ stars are known to be relatively fast rotators (in comparison with sdB stars; Hirsch et al. 2008). The existence of high mass-loss rates in old metal-rich populations is also an important ingredient of one of the most successful hypothesis for modeling the UV-upturn of elliptical galaxies (Yi \& Yoon 2004). If this is so, it is very probable that a fraction of stars in these populations will undergo the helium core flash $(\mathrm{HeCF})$ after departing from the first red giant branch (RGB) - i.e. will be "hot-flashers" as coined by D'Cruz et al. (1996). Finally, in high stellar density enviroments, it is possible that enhanced mass loss might result from star-to-star encounters.

In general, low mass stars undergo the $\mathrm{HeCF}$ at the tip of the RGB. However, Castellani \& Castellani (1993) showed that, if sufficient mass is lost on the RGB, the star can depart from 
the RGB and experience the HeCF while descending the hot white dwarf cooling track. Afterwards D'Cruz et al. (1996) analyzed the frequency of these HeCFs at high temperatures ("Hot Flashers") by assuming different values of the mass-loss efficiency and suggested that this scenario might provide a path for populating the hot end of the HB. Sweigart (1997) noticed the similarity between these hot-flashers and the late helium shell flashes that produce "born-again AGB stars" (Iben 1984) and showed that - as happens in the born-again scenario - the convective zone, generated by the extremely high energy liberation rate $\left(L_{\mathrm{He}} \sim 10^{10} L_{\odot}\right)$ during the flash, reaches the H-rich envelope and engulfs it. As a consequence, much or almost all $\mathrm{H}$ is burned leading to a He-enriched surface abundance. Brown et al. (2001) explored this scenario to explain anomalies in the hot HB of NGC 2808. However, it was not until the work of Cassisi et al. (2003) that simulations including the violent mixing and burning of $\mathrm{H}$ were performed and yields for the abundances of helium, carbon and nitrogen were calculated consistently. All of these works were focused mainly on globular clusters and therefore on low metallicity progenitors. However, counterparts of these hot-flashers are also expected in the Galactic disk. This was considered by Lanz et al. (2004) who assessed the possibility that (field) He-sdB stars could be formed by the hot-flasher scenario. In this work, the authors classified three different clases of hotflashers: (1) early hot-flashers, which experience the HeCF during the evolution at constant luminosity in the HR diagram (after departing from the RGB-tip) and become hot subdwarf stars with standard $\mathrm{H} / \mathrm{He}$ envelopes; (2) late hot-flashers with shallowmixing (SM), which become He-enriched hot subdwarfs due to convective dilution of the envelope (at the low-luminosity, low temperature region of the HR diagram) into deeper regions of the star; and (3) late hot flashers with deep-mixing (DM) in which the $\mathrm{H}$-rich envelope is engulfed and burned in the convective zone generated by the primary HeCF. Due to the numerical difficulties related to the calculation of the violent $\mathrm{H}$ burning that occurs during a deep-mixing event (see Cassisi et al. 2003); Lanz et al. (2004) failed to provide surface abundances in this case. Finally, Ströer et al. $(2005,2007)$ and Hirsch et al. (2008) analyzed the possibility that field $\mathrm{He}-\mathrm{sdO}$ could be generated by this mechanism and concluded that the idea could not be rejected, although some serious discrepancies exist between models and observations. However, the lack of a quantitative study of hot-flasher events and the chemical composition that arise from them for a wide range of metallicities, ensures that comparison with observations remains difficult. We note that only one previous work (Cassisi et al. 2003) has calculated the abundances produced by the deep-mixing event but only for one remnant mass and metallicity $(Z=0.0015)$. On the other hand Lanz et al. (2004) calculated several sequences for solar-like metallicities but no calculation of the final surface abundances for the deepmixing events were presented. The amount of observational data for these stars is increasing rapidly as a byproduct of surveys of faint blue objects such as SDSS and SPY. As concluded by Cassisi et al. (2003) to assess the viability of the hot-flasher scenario, a study of the dependence of the hot-flasher predictions ( $T_{\text {eff }}, g$ and abundances) on the physical details is needed.

The core feature of this article is to present a homogeneous set of simulations of the hot-flasher scenario for a wide range of cases in which the chemical abundances of the models are consistently calculated. In particular we present simulations for 4 different metallicities and different post-RGB remnant masses (which determines the luminosity of the model at the moment of the $\mathrm{HeCF}$ ). The work is organized as follows. In the following section, we discuss the main ingredients of the simulations performed in this work. We then, in Sect. 3, discuss different flavors of hot-flasher and He-enrichment and describe our simulations results under standard assumptions. In Sect. 4, we analyze deviations from the standard assumptions such as possible extramixing at convective boundaries, the effect of chemical gradients, or a possible reduction in convective velocities. We also address the possible effects of element diffusion in the outer layers. In Sect. 5, we discuss the results of our standard sequences and compare them with observations. Finally, in Sect. 6, we present some concluding remarks.

\section{Numerical and physical details}

The sequences presented in this work were calculated with LPCODE, a numerical code for solving the equations of stellar evolution, which was recently used to model the formation of $\mathrm{H}$-deficient post-AGB stars in the born-again scenario (Miller Bertolami et al. 2006; Miller et al. 2007) and, with minor changes, to study global properties of the evolution of low and intermediate stars (Serenelli \& Fukugita 2007). LPCODE is a Henyey-type stellar evolution code designed specifically to compute the formation and evolution of white dwarf stars and described extensively by Althaus et al. (2003, 2005, and references therein). We therefore only briefly refer to the code to mention some particular features and some modifications of special interest to this work.

Radiative opacities are those of OPAL (Iglesias \& Rogers 1996), including opacities for C-rich mixtures as expected for the regions with violent proton burning during a deep-mixing event (Cassisi et al. 2003) complemented at low temperatures with the molecular opacities of Alexander \& Ferguson (1994). In the present work, conductive opacities for the degenerate Hecore were included according to Cassisi et al. (2007). As a consequence, we note that the core masses of our models at the HeCF are slightly lower than in previous works with $\Delta M_{\mathrm{He}-\text { core }} \sim$ $0.006 M_{\odot}$ for all metallicities (see Cassisi et al. 2007). Neutrino emission due to plasma processes was calculated as described by Haft et al. (1994). We used a nuclear network that considers 16 elements and 34 nuclear reactions of the p-p chains, CNO bi-cycle, helium burning, and carbon ignition that are identical to those adopted by Althaus et al. (2005) with the exception of ${ }^{12} \mathrm{C}+\mathrm{p} \rightarrow{ }^{13} \mathrm{~N}+\gamma \rightarrow{ }^{13} \mathrm{C}+\mathrm{e}^{+}+v_{\mathrm{e}}$ and ${ }^{13} \mathrm{C}(\mathrm{p}, \gamma){ }^{14} \mathrm{~N}$, of special interest in this work, which are now taken from Angulo et al. (1999).

In this work, mixing and burning of elements are solved simultaneously in the context of diffusive convective mixing. This is performed by solving the set of equations

$$
\frac{\mathrm{d} \boldsymbol{X}}{\mathrm{d} t}=\left(\frac{\partial \boldsymbol{X}}{\partial t}\right)_{\text {nuc }}+\frac{\partial}{\partial m}\left[\left(4 \pi r^{2} \rho\right)^{2} D \frac{\partial \boldsymbol{X}}{\partial m}\right]
$$

where $\boldsymbol{X}$ is a vector containing the abundances of all considered elements (see Althaus et al. 2003, for details of the numerical procedure). The efficiency of convection (or any other mixing process) is described by adopting the appropriate diffusion coefficient, $D$. In the present work convection was solved (generally, see Sect. 4) with the standard mixing length theory (MLT), adopting a value of $\alpha=1.61$ for the free parameter of the MLT. This value allowed us to reproduce the present luminosity and effective temperature of the sun, $\log T_{\text {eff }}=3.7614$ and $L_{\odot}=3.842 \times 10^{33} \mathrm{erg} \mathrm{s}^{-1}$, at an age of $t=4570 \mathrm{Myr}$, when adopting $Z=0.0164$ and $X=0.714$ in agreement with the $Z / X$ 
Table 1. Initial (ZAMS) values of our standard sequences.

\begin{tabular}{cc}
\hline \hline $\begin{array}{c}\text { Mass at ZAMS } \\
\left(M_{\odot}\right)\end{array}$ & $\begin{array}{c}\text { Initial abundances } \\
(\mathrm{X} / \mathrm{Y} / \mathrm{Z})\end{array}$ \\
\hline 0.88 & $0.769 / 0.230 / 0.001$ \\
0.98 & $0.736 / 0.254 / 0.010$ \\
1.03 & $0.702 / 0.278 / 0.020$ \\
1.04 & $0.668 / 0.302 / 0.030$ \\
\hline
\end{tabular}

value of Grevesse \& Sauval (1998). In the context of the MLT, convection is described by

$D=\frac{\alpha H_{\mathrm{P}} v_{\mathrm{MLT}}}{3}=\frac{\alpha^{4 / 3} H_{\mathrm{P}}}{3}\left[\frac{c g}{\kappa \rho}(1-\beta) \nabla_{\mathrm{ad}}\left(\nabla_{\mathrm{rad}}-\nabla\right)\right]^{1 / 3}$.

Initial (ZAMS) masses for our simulations (Table 1) were chosen to obtain ZAHB ages close to those of globular clusters ( 12.5 Gyr, Salaris \& Weiss 2002). However we do not expect initial mass to play a role in these simulations and the results should therefore be valid for sequences of ages at the moment of the $\mathrm{HeCF}$ similar to that of the disk $(\sim 8 \mathrm{Gyr})^{1}$. Mass loss in LPCODE is now included with the prescription of Schröder \& Cuntz (2005). However, as the details of mass loss in the RGB do not correspond to distinctive features in the models after the star has departed from the RGB, we enhanced mass loss artificially close to the RGB tip to obtain different $\mathrm{H}$-rich envelope masses at the moment of the HeCF. Mass loss was stopped arbitrarily when the star reached $T_{\text {eff }} \sim 12600 \mathrm{~K}$.

The initial helium content of the sequences (which span a wide metallicity range) was chosen to be

$Y=0.23+2.4 \times Z$

allowing comparison with previous works, that have adopted $Y=0.23$ for metal-poor globular clusters, while at the same time is consistent with our adopted solar values and similar to present determinations of the chemical evolution of the galaxy (Flynn 2004; Casagrande et al. 2007).

\section{Description of standard sequences}

We performed simulations of the late hot-flasher scenario in which the surface composition is altered by both dilution and burning of the H-rich envelope. Evolutionary sequences were followed from the ZAMS, through to the hot-flasher event, and to the post horizontal branch evolution. Our standard sequences include neither overshooting at any convective boundary nor any other extra-mixing processes (such as semiconvection). In these sequences, we also neglected the effect of the $\mu$-gradients at the $\mathrm{H}-\mathrm{He}$ transition and convective velocities were those predicted by the MLT. Since our aim is to provide a complete and homogeneous set of observable properties of the predictions of these events, we performed full evolutionary simulations for the different flavors of the hot-flasher scenario and four different metallicities $(Z=0.001,0.01,0.02$ and 0.03$)$, and for He-core flashes at different points of the post-RGB evolution, i.e. at different remnant masses. Initial abundances and masses of these sequences are shown in Table 1.

Before describing of the surface properties of these sequences, we provide a brief description of how H-deficiency is achieved in the simulations.

${ }^{1}$ For example at $Z=0.02$ the mass of the He-core at the HeCF is less than $0.5 \%$ smaller for a higher mass progenitor $\left(1.25 M_{\odot}\right)$ that undergoes the HeCF $\sim 6$ Gyr after the ZAMS.

\subsection{Cases of surface H-depletion}

Late hot-flashers, i.e. those that experience He-enrichment of their stellar surfaces, were classified by Lanz et al. (2004) into "shallow" and "deep" mixing cases. In shallow-mixing (SM) cases, no H-burning occurs and He-enrichment is attained only by dilution of the remaining H-rich envelope into deeper regions. In contrast, in deep-mixing (DM) cases the H-rich envelope is engulfed by He-flash convective zones and $\mathrm{H}$ is burned violently in the hot interior of the star. This picture is not entirely consistent with our results in particular because there are intermediate cases in which, although He-enrichment is due to dilution some inner parts of the H-rich envelope are burnt during the episode. In these cases $L_{\mathrm{H}}$, can be momentaneously as high as $10^{7} L_{\odot}$ but only a fraction of the $\mathrm{H}$-content is finally burnt. Since there is no sharp separation between these intermediate cases and the "shallow mixing" cases of Lanz et al. (2004), we retain the "shallow mixing" denomination for both cases. However, we denote the existence of some important H-burning during the flash by labeling those cases in which H-burning luminosity reached values momentaneously higher than $10^{4} L_{\odot}$ as $\mathrm{SM}^{*}$ (see Table 2). On the other hand, the separation between DM and SM cases is clearly characterized by the development of a violent runaway burning of protons during DM cases and, especially, by the almost complete burning of the original $\mathrm{H}$ content of the remnant. In the following paragraphs, we briefly describe these different cases of late hot-flashers.

Since the evolution of convective zones during all cases of late hot-flashers have some common features, we discuss these first. First, as a consequence of neutrino cooling, the primary $\mathrm{HeCF}$ develops off-center. A growing convective zone develops in the He-core above the point of maximum release of energy, with its upper boundary becoming progressively closer to the $\mathrm{H}-\mathrm{He}$ transition. When the flash is fully developed and the structure of the star changes as a consequence of the sudden energy released by the primary $\mathrm{HeCF}$ - the star is moving to low luminosity and low temperature regions of the HR diagram see Fig. 1 - a very shallow inwardly growing convective zone develops due to opacity and lower surface temperatures of the models. It is this convective zone that ultimately exposes the H-deficient material when it merges with some of the convective zones developed in the interior of the star. This is true for all kinds of late hot-flashers (see Figs. 2-4).

The evolution of the DM episode is shown in Figs. 1 and 2. First, the He-flash starts off-center and similar to those at the tip of the RGB, developing a convective zone whose upper boundary becomes closer to the $\mathrm{H}-\mathrm{He}$ transition as the He-burning luminosity rises. As reported by Sweigart (1997), the entropy barrier (due to previous CNO burning) is insufficiently high because the H-burning shell is almost extinguished at the moment of the flash - and the $\mathrm{HeCF}$ driven convective zone is able to penetrate the H-rich envelope, bringing protons into the C-enriched (a few percent by mass) He-burning convective zone. As a consequence, $\mathrm{H}$ burns producing an extra energy release. At some point, the rate of $\mathrm{H}$-rich material brought down and burnt in the interior is so high that the energy released by H-burning becomes very important and two important events occur. First, due to the rise in the entropy of the material at the location at which protons burn these layers become convectively stable splitting the previous convective zone into two, one powered by the He-flash and the other by H-burning. Second, the energy released by proton burning becomes the main source in driving further expansion the convective region leading to an unstable situation: a further increase in the convective zone produces an 
Table 2. Properties of some of the standard sequences calculated for this work. Fifth, sixth, and seventh column indicate the interval of time between the maximum of $\log \left(L_{\mathrm{He}} / L_{\odot}\right)$ and $\log \left(L_{\mathrm{H}} / L_{\odot}\right)\left({ }^{1} \Delta t\right)$, from the primary He flash to the ZAHB $\left({ }^{2} \Delta t\right)$ and from the ZAHB to the end of the EHB (TAHB; $\left.{ }^{3} \Delta t\right)$. The final three columns provide the total $\mathrm{H}$ content of the sequence just before the He flash $\left({ }^{1} \mathrm{M}_{\mathrm{H}}\right)$, after the primary He core flash $\left({ }^{2} \mathrm{M}_{\mathrm{H}}\right)$ and at the ZAHB $\left({ }^{3} \mathrm{M}_{\mathrm{H}}\right)$.

\begin{tabular}{|c|c|c|c|c|c|c|c|c|c|}
\hline $\begin{array}{c}\text { Final mass } \\
{\left[M_{\odot}\right]}\end{array}$ & $Z_{0}$ & $\begin{array}{c}\text { Maximum } \\
\log \left(L_{\mathrm{He}} / L_{\odot}\right)\end{array}$ & $\begin{array}{c}\text { Maximum } \\
\log \left(L_{\mathrm{H}} / L_{\odot}\right)\end{array}$ & $\begin{array}{l}{ }^{1} \Delta t \\
{[\mathrm{yr}]}\end{array}$ & $\begin{array}{c}{ }^{2} \Delta t \\
{[\mathrm{Myr}]}\end{array}$ & $\begin{array}{c}{ }^{3} \Delta t \\
{[\mathrm{Myr}]}\end{array}$ & $\begin{array}{l}{ }^{1} \mathrm{M}_{\mathrm{H}} \\
{\left[M_{\odot}\right]}\end{array}$ & $\begin{array}{l}{ }^{2} \mathrm{M}_{\mathrm{H}} \\
{\left[M_{\odot}\right]}\end{array}$ & $\begin{array}{l}{ }^{3} \mathrm{M}_{\mathrm{H}} \\
{\left[M_{\odot}\right]}\end{array}$ \\
\hline $0.49145\left(\mathrm{SM}^{*}\right)$ & 0.001 & 10.23 & 7.44 & 9006 & 1.53 & 66.73 & $4.19 \times 10^{-4}$ & $3.21 \times 10^{-4}$ & $3.21 \times 10^{-4}$ \\
\hline $0.49104(\mathrm{DM})$ & 0.001 & 10.22 & 9.24 & 106.78 & 1.55 & 67.33 & $4.17 \times 10^{-4}$ & $2.92 \times 10^{-5}$ & $2.29 \times 10^{-6}$ \\
\hline $0.48545(\mathrm{DM})$ & 0.001 & 10.10 & 10.09 & 7.35 & 1.55 & 65.20 & $4.30 \times 10^{-4}$ & $2.37 \times 10^{-7}$ & $4.16 \times 10^{-8}$ \\
\hline $0.48150(\mathrm{DM})$ & 0.001 & 10.09 & 10.71 & 4.11 & 1.57 & 69.60 & $4.41 \times 10^{-4}$ & $2.10 \times 10^{-6}$ & $2.46 \times 10^{-7}$ \\
\hline $0.47770(\mathrm{SM})$ & 0.01 & 9.83 & 3.58 & 79123 & 1.71 & 71.82 & $2.93 \times 10^{-4}$ & $2.44 \times 10^{-4}$ & $2.44 \times 10^{-4}$ \\
\hline $0.47744\left(\mathrm{SM}^{*}\right)$ & 0.01 & 9.83 & 6.91 & 180471 & 1.66 & 71.96 & $2.93 \times 10^{-4}$ & $1.38 \times 10^{-4}$ & $1.31 \times 10^{-4}$ \\
\hline $0.47725(\mathrm{DM})$ & 0.01 & 9.82 & 9.12 & 2960 & 1.71 & 72.69 & $2.92 \times 10^{-4}$ & $2.33 \times 10^{-6}$ & $1.85 \times 10^{-7}$ \\
\hline $0.46921(\mathrm{DM})$ & 0.01 & 9.66 & 9.91 & 54.7 & 1.71 & 84.69 & $3.06 \times 10^{-4}$ & $6.72 \times 10^{-7}$ & $8.35 \times 10^{-8}$ \\
\hline $0.46644(\mathrm{DM})$ & 0.01 & 9.71 & 9.70 & 44.1 & 1.65 & 78.76 & $3.11 \times 10^{-4}$ & $1.96 \times 10^{-7}$ & $3.78 \times 10^{-8}$ \\
\hline $0.47378(\mathrm{SM})$ & 0.02 & 9.71 & - & - & 1.80 & 73.89 & $2.62 \times 10^{-4}$ & $2.62 \times 10^{-4}$ & $2.61 \times 10^{-4}$ \\
\hline $0.47250\left(\mathrm{SM}^{*}\right)$ & 0.02 & 9.69 & 5.78 & 3053 & 1.80 & 78.99 & $2.54 \times 10^{-4}$ & $9.66 \times 10^{-5}$ & $6.85 \times 10^{-5}$ \\
\hline $0.47112(\mathrm{DM})$ & 0.02 & 9.66 & 9.33 & 706 & 1.82 & 76.48 & $2.55 \times 10^{-4}$ & $8.27 \times 10^{-7}$ & $8.95 \times 10^{-8}$ \\
\hline $0.46410(\mathrm{DM})$ & 0.02 & 9.52 & 10.06 & 135.8 & 1.83 & 88.10 & $2.66 \times 10^{-4}$ & $7.32 \times 10^{-7}$ & $7.91 \times 10^{-8}$ \\
\hline $0.46150(\mathrm{DM})$ & 0.02 & 9.60 & 10.25 & 132.4 & 1.74 & 81.75 & $2.70 \times 10^{-4}$ & $2.01 \times 10^{-7}$ & $3.48 \times 10^{-8}$ \\
\hline $0.46521(\mathrm{SM})$ & 0.03 & 9.45 & - & - & 1.87 & 83.33 & $2.67 \times 10^{-4}$ & $2.67 \times 10^{-4}$ & $2.56 \times 10^{-4}$ \\
\hline $0.46470(\mathrm{SM})$ & 0.03 & 9.45 & 2.67 & 78688 & 1.89 & 79.70 & $2.48 \times 10^{-4}$ & $1.70 \times 10^{-4}$ & $3.44 \times 10^{-5}$ \\
\hline $0.46367\left(\mathrm{SM}^{*}\right)$ & 0.03 & 9.43 & 5.80 & 17248 & 1.90 & 80.76 & $2.49 \times 10^{-4}$ & $7.23 \times 10^{-5}$ & $1.16 \times 10^{-5}$ \\
\hline $0.46282(\mathrm{DM})$ & 0.03 & 9.41 & 9.00 & 10926 & 1.93 & 81.47 & $2.49 \times 10^{-4}$ & $4.41 \times 10^{-6}$ & $3.28 \times 10^{-7}$ \\
\hline $0.45660(\mathrm{DM})$ & 0.03 & 9.27 & 9.80 & 975 & 2.00 & 87.76 & $2.49 \times 10^{-4}$ & $7.05 \times 10^{-7}$ & $5.52 \times 10^{-8}$ \\
\hline $0.45234(\mathrm{DM})$ & 0.03 & 9.31 & 9.49 & 1055 & 1.94 & 93.93 & $2.58 \times 10^{-4}$ & $5.64 \times 10^{-7}$ & $6.47 \times 10^{-8}$ \\
\hline
\end{tabular}

increase in the power released by H-burning, which leads to a further outward excursion of the convective boundary. This produces a $\mathrm{H}$-flash in which almost all $\mathrm{H}$-content of the remnant is rapidly burnt, an episode that is also characterized by a rapid outward excursion of the outer boundary of the convective zone. This additional growth in the convective zone can be clearly seen in Fig. 2 about 1 year after the primary HeCF. During the episode, the star moves to the low luminosity, low temperature region of the HR diagram (see Fig. 1), where a very shallow outer convective zone develops. This convective zone becomes progressively deeper and ultimately, some years after the primary $\mathrm{HeCF}$, merges with the inner convective zones and the surface of the star becomes H-deficient (Fig. 2). After this, the star proceeds through several secondary helium flashes, like in the case of "canonical helium core flashes" (i.e. at the RGB tip), before finally settling at the ZAHB as an extremely H-deficient $\operatorname{star}\left(X_{\mathrm{H}} \sim 10^{-4}-10^{-6}\right)$. The hydrogen content decreases from a few $10^{-4} M_{\odot}$ to usually less than $10^{-6} M_{\odot}$ (see Table 2 ) after the primary helium flash.

If the flash occurs when the H-burning shell is somewhat more luminous (a $\mathrm{SM}^{*}$ case), then the convective zone may come into contact with the inner part (with low $\mathrm{H}$ content) of the H-rich envelope, but the material that is brought downwards is never sufficient for the H-flash to develop. This is shown in Fig. 3 (see also the middle panel of Fig. 1). Then only a tiny fraction of the envelope is burned - e.g. the total H-content of the star changes, after the entire series of $\mathrm{HeCFs}$, from $2.5 \times 10^{-4}$ to $6.9 \times 10^{-5} M_{\odot}$, in the case shown in Fig. 3, and in the $Z=0.001 \mathrm{SM}^{*}$ case of Table 2 only about $\sim 10^{-4} M_{\odot}$ is burnt during the primary HeCF. Afterwards, the outer boundary of the flash driven convective zone penetrates slowly, diluting the inner regions of the $\mathrm{H}$-rich envelope until it finally merges with the inwardly growing surface convective zone, turning the star into a hydrogen deficient star with low $\mathrm{H}$ content in the envelope $(X$ of $10^{-2}$ to $10^{-3}$ by mass fraction, see Table 3 ).
Finally, if the flash occurs when the H-burning shell is even more active (see Fig. 4, and also the top panel of Fig. 1), then the convective zone does not come into contact with the H-rich envelope, due to the entropy, barrier and no $\mathrm{H}$ is burned (this is the case originally referred to as "shallow mixing" by Lanz et al. 2004). After the He-flash, a small tail of the HeCF convective zone remains for many thousand years moving outwards in mass. The convective zone reaches the $\mathrm{H}$-rich envelope and dilutes its inner parts into the $\mathrm{H}$-free interior. Finally, it merges with the inwardly growing surface convective zone, producing a mild H-deficiency in the stellar surface.

Results of numerical simulations of all these events are presented in Table 2, where the timescales and $\mathrm{H}$-content at different stages are given.

\subsection{Sequences in the mass-metallicity plane}

In Fig. 5, we show the standard sequences calculated for this work in the mass-metallicity ${ }^{2}$ plane. As previously mentioned the masses of hot-flasher remnants are slightly lower than in previous works due to the use of the new conductive opacities presented by Cassisi et al. (2007).

Our results support the claim by Lanz et al. (2004) that shallow-mixing is more important in sequences of high metallicity (see Fig. 5). While shallow-mixing accounts for only $4 \%$ of the remnant mass range of late hot-flashers in lower metallicity sequences $(Z=0.001$ and $Z=0.01)$, it corresponds to $14-17 \%$ of the remnant's mass range in the $Z=0.02$ and $Z=0.03$ sequences. The remnant mass range for hot-flashers is found to be

\footnotetext{
2 By metallicity we mean the original metal (ZAMS) content of the star. During this work we choose to label the sequences with their final mass instead of the mass loss rate - as done in previous works - because it is the final mass of the remnant, and not the particular way in which mass is lost, which plays an important role in the development of the different kinds of hot-flashers.
} 


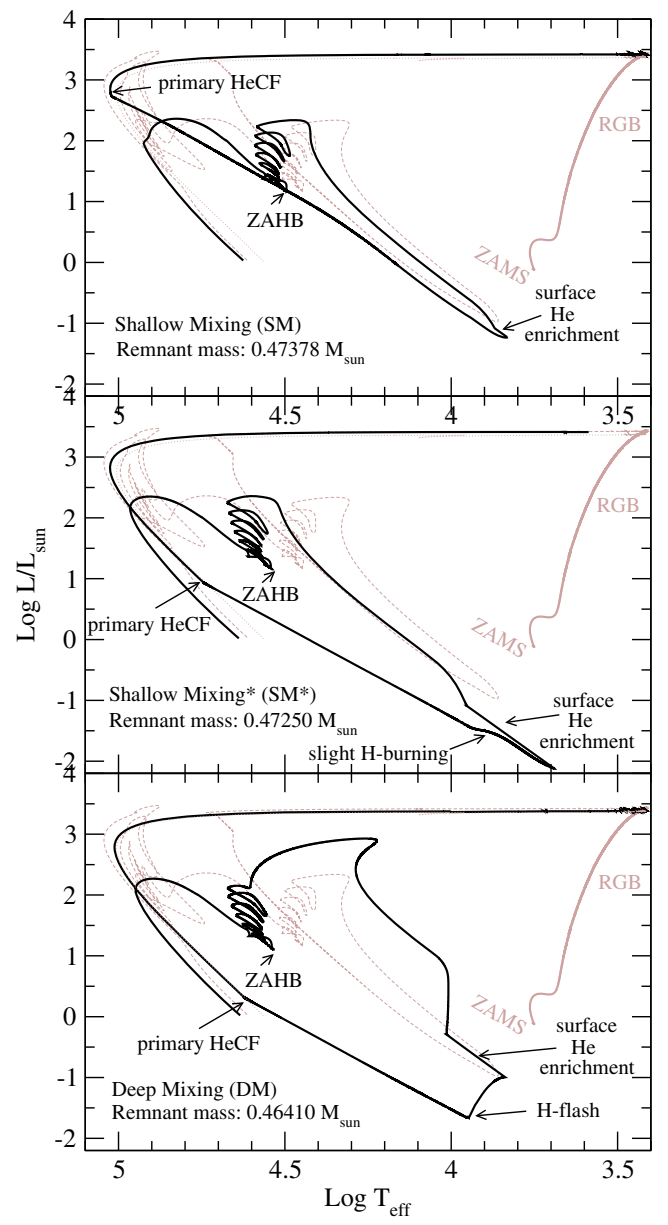

Fig. 1. HR diagrams for the three kinds of late hot-flashers described in this work. Sequences correspond to $Z=0.02$. For comparison, an early hot-flasher sequence of $0.47426 M_{\odot}$ that finishes with normal $\mathrm{H}$ abundances (broken lines), and a HeCF-manque sequence of $0.4607 M_{\odot}$ that ends as a He core white dwarf (dotted line) are also plotted (color figure only available in the electronic version).

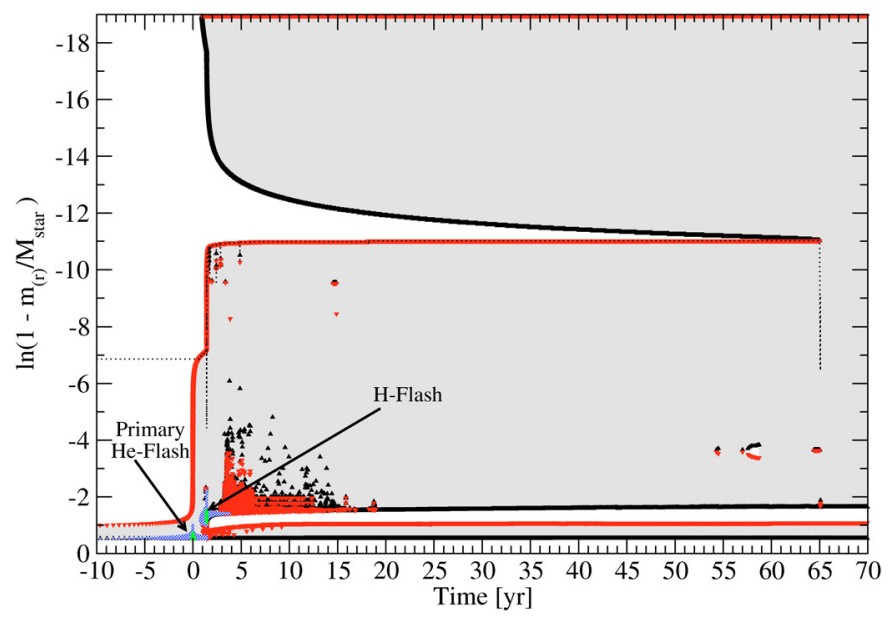

Fig. 2. Kippenhahn diagram of a deep-mixing case. The case plotted corresponds to a $0.47017 M_{\odot}$ sequence of initially $Z=0.02$. Red (black) points mark the upper (lower) convective boundaries of each model. Main convective zones have been shaded for clarity. Blue (green) zones mark the regions in which energy generation per unit mass is above $\epsilon>10^{7} \mathrm{erg} \mathrm{g}^{-1} \mathrm{~s}^{-1}\left(\epsilon>10^{9} \mathrm{erg} \mathrm{g}^{-1} \mathrm{~s}^{-1}\right)$. Dotted line marks the location of the inner boundary of the H-rich envelope, defined by $X \sim 0.001$ (color figure only available in the electronic version).

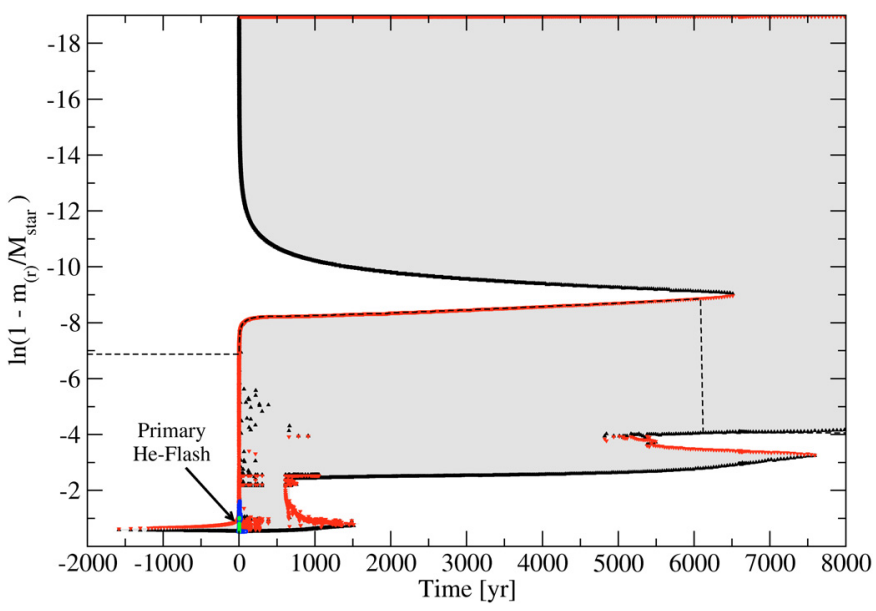

Fig. 3. Kippenhahn diagram of shallow-mixing with slight CNO burning $\left(\mathrm{SM}^{*}\right)$. References are similar to those of Fig. 2, (color figure only available in the electronic version).

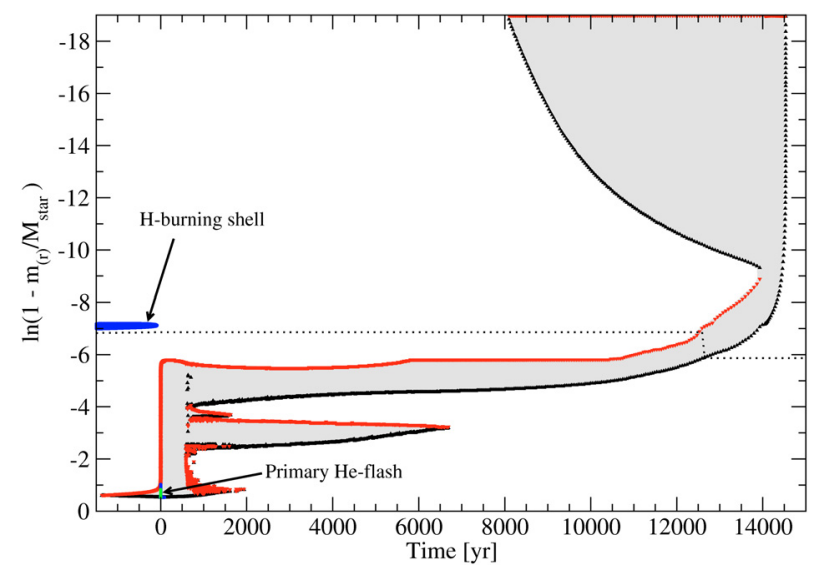

Fig. 4. Kippenhahn diagram of shallow-mixing without slight $\mathrm{CNO}$ burning. References are similar to those of Fig. 2, (color figure only available in the electronic version).

slightly dependent on metallicity, varying from $\Delta M \sim 0.014 M_{\odot}$ for $Z=0.03$ to $\Delta M \sim 0.0105 M_{\odot}$ for $Z=0.001$, although this increase is mainly due to the increase in the SM mass range, while the DM mass range is almost constant. It is not easy to translate this result into a mass range for initial masses because this depends strongly on the mass-loss prescription or mechanism.

\subsection{Surface properties}

In Table 3, we present the surface abundances of our simulations for selected sequences. We first note that, while DM episodes lead to surface hydrogen abundances preferentially between $10^{-5}$ and $10^{-6}$ by mass fraction (although they can be as high as a few $10^{-4}$ in some simulations) the $\mathrm{SM} / \mathrm{SM}^{*}$ episodes offer a wide range of surface $\mathrm{H}$ abundances ranging from almost solar to a few $10^{-3}$ by mass fraction. The $\mathrm{SM} / \mathrm{SM}^{*}$ scenarios therefore provide the intermediate abundances needed by Unglaub (2005) to reproduce $\mathrm{He}$-sdB/He-sdO surface abundances in the light of calculations that include both the effects of diffusion and weak mass loss rates $\left(\dot{M} \lesssim 10^{-13} M_{\odot} / \mathrm{yr}\right)$. This result is quite interesting because some effects not included in these simulations (particularly the effect of the $\nabla \mu$-barrier) may increase the frequency of SM to DM episodes due to the introduction of an extra 
Table 3. Surface abundances (by mass fractions) of some of the sequences calculated in this work.

\begin{tabular}{cccccccc}
\hline \hline Final mass $\left[M_{\odot}\right]$ & $Z_{0}$ & $\mathrm{H}$ & $\mathrm{He}$ & ${ }^{12} \mathrm{C}$ & ${ }^{13} \mathrm{C}$ & $\mathrm{N}$ & $\mathrm{O}$ \\
\hline $0.49145\left(\mathrm{SM}^{*}\right)$ & 0.001 & 0.2137 & 0.7475 & 0.0365 & $1.57 \times 10^{-6}$ & $1.22 \times 10^{-4}$ & $1.52 \times 10^{-3}$ \\
$0.49104(\mathrm{DM})$ & 0.001 & $2.42 \times 10^{-4}$ & 0.9450 & 0.0267 & $7.47 \times 10^{-3}$ & 0.0106 & $4.77 \times 10^{-5}$ \\
$0.48545(\mathrm{DM})$ & 0.001 & $4.60 \times 10^{-6}$ & 0.9524 & 0.0264 & $7.73 \times 10^{-3}$ & 0.0134 & $4.53 \times 10^{-5}$ \\
$0.48150(\mathrm{DM})$ & 0.001 & $2.41 \times 10^{-6}$ & 0.9666 & 0.0107 & $5.79 \times 10^{-3}$ & 0.0185 & $3.79 \times 10^{-5}$ \\
$0.47770(\mathrm{SM})$ & 0.01 & 0.0780 & 0.8682 & 0.0426 & $6.26 \times 10^{-6}$ & $1.30 \times 10^{-3}$ & $6.84 \times 10^{-4}$ \\
$0.47744\left(\mathrm{SM}^{*}\right)$ & 0.01 & 0.0261 & 0.8996 & 0.0642 & $2.36 \times 10^{-6}$ & $6.59 \times 10^{-4}$ & $7.43 \times 10^{-4}$ \\
$0.47725(\mathrm{DM})$ & 0.01 & $2.27 \times 10^{-5}$ & 0.9464 & 0.0382 & $6.12 \times 10^{-3}$ & $4.35 \times 10^{-3}$ & $2.94 \times 10^{-4}$ \\
$0.46921(\mathrm{DM})$ & 0.01 & $1.02 \times 10^{-5}$ & 0.9560 & 0.0211 & $6.13 \times 10^{-3}$ & 0.0128 & $2.92 \times 10^{-4}$ \\
$0.46644(\mathrm{DM})$ & 0.01 & $4.61 \times 10^{-6}$ & 0.9592 & 0.0193 & $5.52 \times 10^{-3}$ & 0.0122 & $3.16 \times 10^{-4}$ \\
$0.47378(\mathrm{SM})$ & 0.02 & 0.3822 & 0.5978 & $1.23 \times 10^{-3}$ & $8.96 \times 10^{-5}$ & $7.57 \times 10^{-3}$ & $4.69 \times 10^{-3}$ \\
$0.47250(\mathrm{SM})$ & 0.02 & $9.56 \times 10^{-3}$ & 0.9273 & 0.0425 & $1.93 \times 10^{-5}$ & $2.94 \times 10^{-3}$ & $8.64 \times 10^{-4}$ \\
$0.47112(\mathrm{DM})$ & 0.02 & $9.05 \times 10^{-6}$ & 0.9389 & 0.0420 & $4.27 \times 10^{-3}$ & $4.88 \times 10^{-3}$ & $6.96 \times 10^{-4}$ \\
$0.46410(\mathrm{DM})$ & 0.02 & $1.05 \times 10^{-5}$ & 0.9460 & 0.0273 & $6.41 \times 10^{-3}$ & 0.0120 & $7.680 \times 10^{-4}$ \\
$0.46150(\mathrm{DM})$ & 0.02 & $4.66 \times 10^{-6}$ & 0.9480 & 0.0263 & $5.67 \times 10^{-3}$ & 0.0116 & $1.01 \times 10^{-3}$ \\
$0.46521(\mathrm{SM})$ & 0.03 & 0.2227 & 0.7271 & 0.0197 & $7.79 \times 10^{-5}$ & $8.09 \times 10^{-3}$ & $5.18 \times 10^{-3}$ \\
$0.46470(\mathrm{SM})$ & 0.03 & $4.71 \times 10^{-3}$ & 0.9239 & 0.0380 & $4.12 \times 10^{-4}$ & $6.10 \times 10^{-3}$ & $1.58 \times 10^{-3}$ \\
$0.46367(\mathrm{SM})$ & 0.03 & $1.63 \times 10^{-3}$ & 0.9249 & 0.0390 & $3.25 \times 10^{-3}$ & $6.48 \times 10^{-3}$ & $1.62 \times 10^{-3}$ \\
$0.46282(\mathrm{DM})$ & 0.03 & $7.48 \times 10^{-6}$ & 0.9333 & 0.0420 & $1.74 \times 10^{-3}$ & $7.04 \times 10^{-3}$ & $1.55 \times 10^{-3}$ \\
$0.45660(\mathrm{DM})$ & 0.03 & $9.22 \times 10^{-6}$ & 0.9382 & 0.0341 & $4.09 \times 10^{-3}$ & 0.0103 & $1.67 \times 10^{-3}$ \\
$0.45234(\mathrm{DM})$ & 0.03 & $1.94 \times 10^{-5}$ & 0.9411 & 0.0273 & $4.76 \times 10^{-3}$ & 0.0134 & $2.14 \times 10^{-3}$ \\
\hline
\end{tabular}

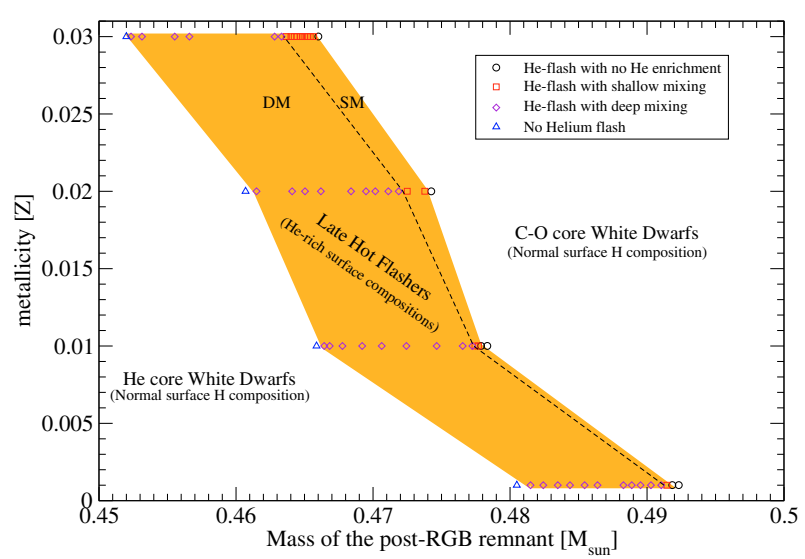

Fig. 5. Mass and metallicity of the standard sequences calculated for this work. The orange zone indicates the sequences that undergo a change in their surface abundances as a consequence of mixing and/or burning of the H-rich envelope. Dashed line marks the separation between DM and SM cases (color figure only available in the electronic version).

barrier against the penetration of the $\mathrm{HeCF}$ convective zone into the H-rich envelope (see Sect. 4).

For the other element abundances, some trends are evident for the DM cases (see Table 3 ). In particular, ${ }^{12} \mathrm{C}$ decreases by a factor of 2 from earlier (i.e. higher mass remnants) to later cases (i.e. lower mass remnants) ${ }^{3}$. This is because, for later DM episodes, the $\mathrm{H}$-flash and the subsequent splitting of the convective zone occur more rapidly than in earlier DM cases (see ${ }^{1} \Delta t$ values in Table 2) and less ${ }^{12} \mathrm{C}$ is therefore created by the time of the $\mathrm{H}$-flash. When the convective zones split, the increase in ${ }^{12} \mathrm{C}$ inside the (inner) He-flash driven convective zone does not affect the final surface abundances. On the other hand, ${ }^{13} \mathrm{C}$ shows no definite trend and is almost constant for a given metallicity, and decreases slightly when metallicity is increased. In Table 3, we

\footnotetext{
3 This definition of "later" and "earlier" does not refer to the time between ZAMS and HeCF but to the location in the HR-diagram in which the flash takes place.
}

can see that ${ }^{14} \mathrm{~N}$ clearly increases for later deep-mixing episodes (for a given metallicity). This can be understood easily as a consequence of the earlier development, in those cases, of the $\mathrm{H}$-flash. Since there is a lower amount of ${ }^{12} \mathrm{C}$ in the convective zone when both splitting occurs and H-burning develops, and the $\mathrm{H}$ mass engulfed is almost constant (see Table 2), then there are more protons per each ${ }^{12} \mathrm{C}$ nuclei and more ${ }^{14} \mathrm{~N}$ is therefore formed. Then, at a given metallicity, the ratio of $\mathrm{C} / \mathrm{N}$ can vary by more than a factor of two, depending on the moment of the post-RGB evolution at which the deep mixing occurs. This is interesting in view of the two classes of He-sdO stars $(\mathrm{N}$ - and C- rich) presented by Ströer et al. (2007). The dependence of the $\mathrm{C} / \mathrm{N}$ ratio as a function of initial metallicity indicates that the lower the metallicity, the higher the $\mathrm{N}$ abundance. However, no clear correlation appears to be present between the location of our sequences with higher N/C ratios and the N-rich class of Ströer et al. (2007, see Fig. 6).

In the case of shallow-mixing events, ${ }^{13} \mathrm{C}$ is orders of magnitude lower than for DM because no significant (if any) amount of $\mathrm{H}$ burns during the flash. At first, it may appear strange that $\mathrm{N}$ abundances in some cases, do not differ significantly (although lower) from those of DM episodes. However, during SM events, the material dredged up to the surface corresponds to material previously processed by the CNO cycle in the RGB, which transforms almost all primordial $\mathrm{C}$ and $\mathrm{O}$ into $\mathrm{N}$. In this case, the $\mathrm{N}$ present in the surface is therefore strongly dependent on the initial metallicity of the stars. This explains why the $\mathrm{N}$ abundance of SM events in Table 3 show a strong dependence on $Z_{0}$. The final ${ }^{12} \mathrm{C}$ abundances in SM depends mainly on the interplay between the enormous amount of ${ }^{12} \mathrm{C}$ created by the primary $\mathrm{He}$ core flash and the intensity of the dilution process when the inner and outer convective zones merge.

As noted by Ströer et al. (2007), although the tracks shown in Fig. 6 pass close to the location of He-sdO stars derived by Ströer et al. $(2005,2007)$, their distribution in the $g-T_{\text {eff }}$ diagram does not correspond to the one expected from the simulations. From the point of view of the models, stars should cluster towards the location of the EHB, $4.5 \lesssim \log T_{\text {eff }} \lesssim 4.65$ and $5.9 \lesssim \log g \lesssim 6.2$, because the evolution from the primary $\mathrm{HeCF}$ to the settlement 
Table 4. Comparison of characteristics of DM sequences of this work with the DM sequence of Cassisi et al. (2003).

\begin{tabular}{lccc}
\hline \hline & This work & This work & Cassisi et al. \\
\hline Mass $\left[M_{\odot}\right]$ & 0.49104 & 0.48545 & 0.49125 \\
$Z_{0}$ & 0.001 & 0.001 & 0.0015 \\
${ }^{1} \Delta t$ & $107 \mathrm{~d}$ & $7.35 \mathrm{~d}$ & $150 \mathrm{~d}$ \\
$\max . L_{\mathrm{He}} / L_{\odot}$ & $1.66 \times 10^{10}$ & $1.26 \times 10^{10}$ & $3.7 \times 10^{10}$ \\
$\max . L_{\mathrm{H}} / L_{\odot}$ & $1.74 \times 10^{9}$ & $1.23 \times 10^{10}$ & $8.2 \times 10^{9}$ \\
$\mathrm{M}_{\mathrm{H}}$ burnt $\left[M_{\odot}\right]$ & $3.88 \times 10^{-4}$ & $4.30 \times 10^{-4}$ & $3.7 \times 10^{-4}$ \\
surf. H & $2.4 \times 10^{-4}$ & $4.6 \times 10^{-6}$ & $4 \times 10^{-4}$ \\
surf. He & 0.945 & 0.952 & 0.96 \\
surf. C & 0.0342 & 0.0341 & 0.029 \\
surf. N & 0.0106 & 0.0134 & 0.007 \\
surf. O & $4.8 \times 10^{-5}$ & $4.5 \times 10^{-5}$ & $3.5 \times 10^{-5}$ \\
\hline
\end{tabular}

in the ZAHB is far more rapid (occuring in less than $2 \times 10^{6} \mathrm{yr}$ ) than the He-core burning stage $\left(65-90 \times 10^{6} \mathrm{yr}\right.$, see Table 2$)$. This clustering of He-sdO stars is not observed in the inferred $g-T_{\text {eff }}$ values of real stars (see Fig. 6). A possible explanation for this discrepancy may be that helium-rich atmospheres become hydrogen-rich, due to diffusion processes, in a timescale shorter than the duration of the He-core burning stage (EHB). In Sect. 4.4, we show that such a conversion is, in fact, possible. According to this scenario, late hot-flashers have a He-rich surface composition only during the evolution towards the EHB and then become stars with hydrogen dominated atmospheres. This implies that He-sdO stars evolve into hydrogen-rich $\mathrm{sdB} / \mathrm{sdO}$ stars, so that no clustering of $\mathrm{He}-\mathrm{sdO}$ stars around the EHB is expected. This scenario is discussed in Sects. 4.4 and 5.

When comparing theoretical predictions with the results of Ströer et al. (2007), we recall that surface properties in that work were obtained by adopting $\mathrm{H} / \mathrm{He}$ atmospheres, which was found by Lanz et al. (2004) (who adopted $\mathrm{H} / \mathrm{He} / \mathrm{C}$ atmospheres in their analyzes) to be a possible cause of systematic effects. The effective temperature inferred in real stars may also be systematically overestimated due to line-blanketing effects (see Ströer et al. 2007).

Finally, we note that the surface characteristics of our solar metallicity DM sequences agree closely with the values inferred by Lanz et al. (2004) for PG1544+488. This result clearly confirms the statement of Lanz et al. (2004) that this object could be the result of a DM event. Our results, also, confirm that the surface abundances derived by these authors for JL87 agree with those predicted by the SM kind of hot-flasher. In contrast, no sequence presented abundances close to those of LB1766, confirming that this object may have had a very different history.

\subsection{Comparison with previous works}

We compare our results with the very few consistent simulations available in the literature. In the DM, we compare the 0.49125 sequence of Cassisi et al. (2003), for which the He-core flash ocurrs at $\log L / L_{\odot} \sim 0.79$ (a relatively "early" deep-mixing episode in the terminology of Sect. 3.2) with the most similar models in our grid. This is shown in Table 4

where the results for the $0.48545 M_{\odot}$ and $0.49104 M_{\odot}$ (with $Z=0.001$ ) sequences of this work are compared with the DM sequence of Cassisi et al. (2003). We note in particular the good agreement between the earlier deep-mixing sequence $\left(0.49104 M_{\odot}\right)$ and Cassisi et al. (2003) values, regardless of the different metallicity and mass in both sequences.

For the SM case, the only available sequence is one of solar metallicity discussed by Lanz et al. (2004), which we can compare with our $0.47378 M_{\odot}, Z=0.02 \mathrm{SM}$ sequence. In their sequence the shallow-mixing event (the merging of the inner and outer convective zones) occurs about $24000 \mathrm{yr}$ after the primary He-core flash compared with the $14000 \mathrm{yr}$ in our sequence (see Fig. 3). The surface abundances in their sequence are $\mathrm{He} / \mathrm{C} / \mathrm{N}=0.5 / 0.008 / 0.005$ qualitatively similar to ours $(\mathrm{He} / \mathrm{C} / \mathrm{N}=0.6 / 0.001 / 0.008)$. We can therefore conclude that there is qualitative agreement between Lanz et al. (2004) and our results.

\section{Alternative sequences}

In this section we discuss the effect of some deviations from the standard assumptions made in the previous section. In particular, we address the effect of possible extra-mixing at convective boundaries, different mixing velocities, and chemical gradients. Finally, we address the effect of diffusion in the outer layers of the standard sequences.

\subsection{Effect of the location of proton burning (altering D)}

The calculation of 1D-stellar evolution models through the $\mathrm{H}$-flash in previous simulations (Sect. 3) were performed within the picture of diffusive convective mixing. This approach was used in the past for similar situations like late helium-shell flashes (i.e. born again episodes) experienced by post-AGB stars at the beginning of their white dwarf stage (e.g. Iben \& Mac Donald 1995). This picture, which implies that material is homogeneously mixed in each mass shell, is very probably far from reality (see Woodward et al. 2008, for a discussion of this issue) in which convection appears to be an advection process. We now analyze how deep-mixing episodes can be altered if the location of maximum energy generation is displaced from the predictions of MLT plus diffusive mixing. This is done by altering the value of the convective diffusion coefficient $D$ in the mixing equations. We note, however, that because we still assume horizontal homogeneity, these results can only be considered poor approximations of the problem ${ }^{4}$.

Surface abundances in simulations with reduced mixing efficiencies are shown in Table 5. Our results are qualitatively consistent with the preliminary results quoted by Cassisi et al. (2003). They indicate, however, that a reduction in $D$ of only two orders of magnitude is sufficient to produce an increase in the residual amount of $\mathrm{H}$ after a DM episode of about an order of magnitude. In view of the results of Unglaub (2005), it is interesting to note that a reduction in $D$ of a factor of between 1000 and 10000 , produces $\mathrm{H}$-abundances after the DM episode that would finally convert He-sdO stars into He-deficient sdB stars by gravitational settling on the EHB, even in the presence of weak winds $\left(\dot{M} \sim 10^{-13} M_{\odot} /\right.$ yr $)$. If major proton burning is shifted deeper into the star $(D \times 10)$ then the extra energy released by proton burning is locally less relevant - in comparison with the local flux due to the primary He-flash - and the violent proton burning is never sufficient to drive convection further out. As a consequence, the "runaway" burning of protons does not develop and, as can be seen from Table 5, far higher $\mathrm{H}$ abundances are left than in the standard case. The H-abundance after the primary He-flash in this case, may also lead to the conversion of He-sdO stars into He-deficient sdB stars, according to Unglaub (2005).

\footnotetext{
4 Horizontal inhomogeneities in ${ }^{12} \mathrm{C},{ }^{13} \mathrm{C}$ and $\mathrm{H}$ would certainly affect the final ${ }^{14} \mathrm{~N}$ yield. This is exactly the situation expected during the hydrogen flash (see Woodward et al. 2008).
} 


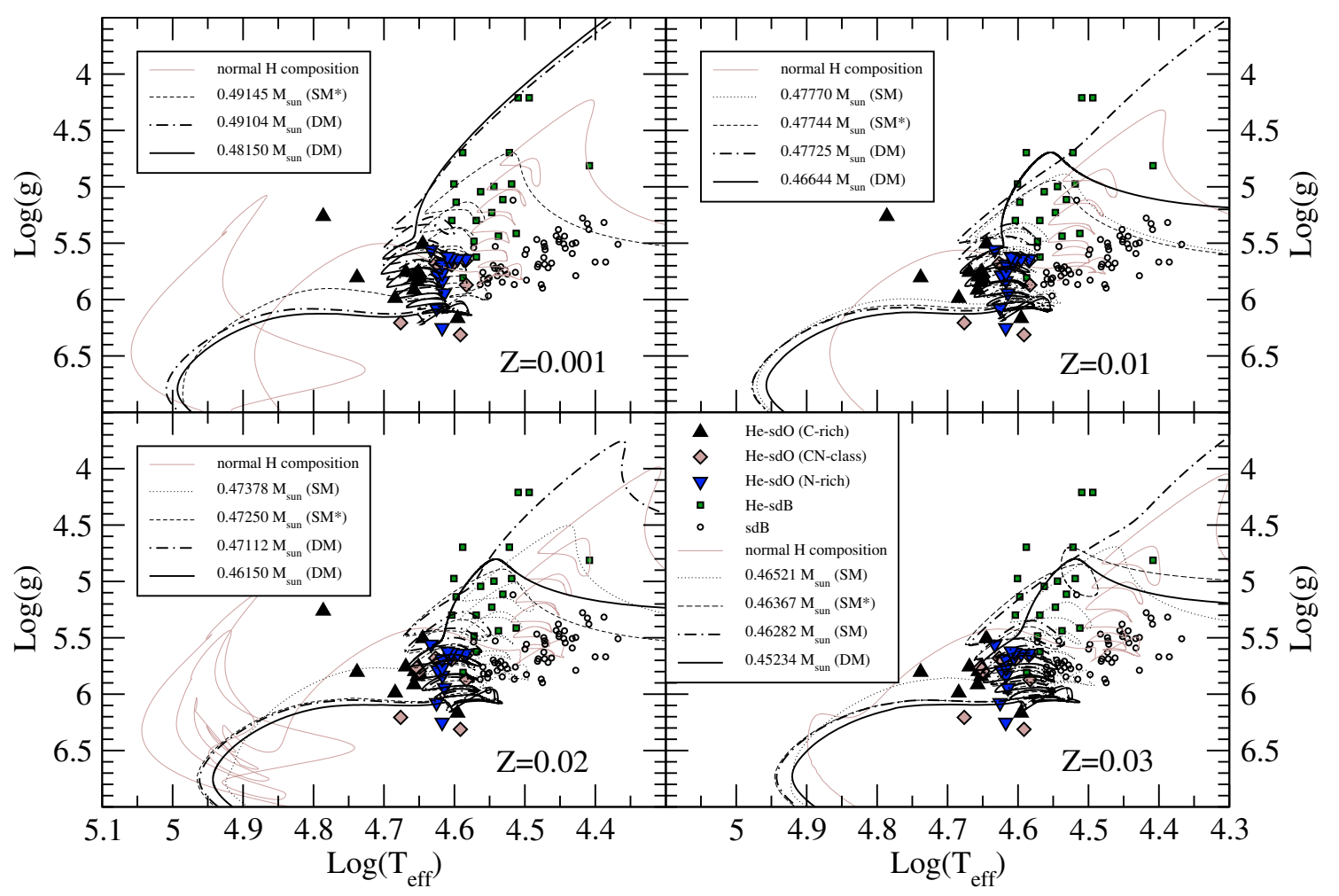

Fig. 6. Values of $T_{\text {eff }}$ and $g$ of our sequences for different metallicities. Values inferred for He-sdO and sdB stars are from by Ströer et al. (2007); Lisker et al. (2005), respectively. He-sdB data is taken from Jeffery (2008) (color figure only available in the electronic version).

Table 5. Surface abundances (by mass fractions) of the sequences calculated in this work with different mixing efficiencies. All sequences correspond to models with a final mass equal to $0.48545 M_{\odot}$ and $Z=0.001{ }^{\dagger}$ This sequence did not undergo the H-flash, see text.

\begin{tabular}{lcccccccc}
\hline \hline Diff. Coef. & $\mathrm{H}$ & $\mathrm{He}$ & ${ }^{12} \mathrm{C}$ & ${ }^{13} \mathrm{C}$ & $\mathrm{N}$ & $\mathrm{O}$ & ${ }^{2} \mathrm{M}_{\mathrm{H}}$ & ${ }^{3} \mathrm{M}_{\mathrm{H}}$ \\
\hline${ }^{\dagger} D_{\mathrm{MLT}} \times 10$ & $1.03 \times 10^{-3}$ & 0.9022 & 0.0942 & $2.09 \times 10^{-6}$ & $1.08 \times 10^{-6}$ & $4.09 \times 10^{-4}$ & $1.42 \times 10^{-5}$ & $8.12 \times 10^{-6}$ \\
$D_{\mathrm{MLT}} \times 3$ & $1.71 \times 10^{-6}$ & 0.9514 & 0.0283 & $7.99 \times 10^{-3}$ & 0.0118 & $5.13 \times 10^{-5}$ & $8.18 \times 10^{-8}$ & $1.54 \times 10^{-8}$ \\
$D_{\mathrm{MLT}}$ & $4.60 \times 10^{-6}$ & 0.9524 & 0.0264 & $7.73 \times 10^{-3}$ & 0.0134 & $4.53 \times 10^{-5}$ & $2.37 \times 10^{-7}$ & $4.16 \times 10^{-8}$ \\
$D_{\mathrm{MLT}} / 10$ & $1.66 \times 10^{-5}$ & 0.9532 & 0.0291 & $7.50 \times 10^{-3}$ & $9.71 \times 10^{-3}$ & $3.62 \times 10^{-5}$ & $7.97 \times 10^{-7}$ & $1.49 \times 10^{-7}$ \\
$D_{\mathrm{MLT}} / 100$ & $4.22 \times 10^{-5}$ & 0.9506 & 0.0328 & $7.66 \times 10^{-3}$ & $8.49 \times 10^{-3}$ & $3.18 \times 10^{-5}$ & $1.95 \times 10^{-6}$ & $3.72 \times 10^{-7}$ \\
$D_{\mathrm{MLT}} / 1000$ & $4.57 \times 10^{-4}$ & 0.9515 & 0.0327 & $6.89 \times 10^{-3}$ & $7.99 \times 10^{-3}$ & $3.06 \times 10^{-5}$ & $2.49 \times 10^{-5}$ & $3.95 \times 10^{-6}$ \\
$D_{\mathrm{MLT}} / 10000$ & 0.0117 & 0.9455 & 0.0376 & $2.53 \times 10^{-3}$ & $1.87 \times 10^{-3}$ & $3.35 \times 10^{-5}$ & $2.27 \times 10^{-4}$ & $7.42 \times 10^{-5}$ \\
\hline
\end{tabular}

We note also that a feature of the absence of the runaway proton burning ( $\mathrm{H}$-flash) is that the convective zone developed by the primary $\mathrm{HeCF}$ never splits and the surface ${ }^{12} \mathrm{C}$ abundance continues to increase until the convective zone merges with the outer convection. In this case, the final ${ }^{12} \mathrm{C}$ is much larger than the cases in which the H-flash develops.

\subsection{Effect of extra-mixing at convective boundaries}

To analyze the effects of possible extra-mixing episodes, we included extra-mixing zones at each convective boundary following the prescription of Herwig et al. (1997). We therefore allowed extra-mixing episodes by considering that the mixing velocities decay exponentially beyond each convective boundary. The diffusion coefficient beyond the formal convective boundary was then given by

$D_{\mathrm{OV}}(z)=D_{0} \exp \left(\frac{-2 z}{f H_{\mathrm{P}}}\right)$ where $D_{0}$ is the diffusion coefficient of unstable regions close to the convective boundary and $z$ is the geometric distance from the edge of the convective boundary. We performed simulations with three different values of the free parameter $f(f=0.005$, 0.01 and 0.1 ) around the value inferred from the width of the upper main sequence $(f \sim 0.016)$ and oxygen abundances of PG1159 stars (Herwig 2000).

Results of these numerical experiments are shown in Table 6. In the case of $f=0.005$, the final surface abundances are close to those of the standard simulations in which no extra-mixing was considered. For larger extra-mixing efficiencies H-burning becomes more efficient and for values beyond $f=0.01$ almost no $\mathrm{H}$ remains in the star after the $\mathrm{H}$-flash. For values of $f \lesssim 0.01$, the evolutionary timescales $\left({ }^{1} \Delta t,{ }^{2} \Delta t,{ }^{3} \Delta t\right.$; see Table 2 for the definition) are unaffected and are similar to the standard case (see Table 2). In contrast, for the $f=0.1$ case, the time interval between flashes is significantly reduced (46 d) and the EHB evolution is somewhat enlarged by the appearance of breathing pulse instabilities at the end of the EHB (212 Myr). The lower 
$\mathrm{N}$ abundance in the $f=0.1$ case is because the $\mathrm{H}$ is mixed with a larger mass of ${ }^{12} \mathrm{C}$ reducing the amount of protons per carbon nuclei.

\subsection{Effect of chemical gradients $(\nabla \mu)$}

Miller Bertolami et al. (2006) recently showed that chemical gradients $(\nabla \mu)$ can affect the development of the rapid ingestion of protons into convective zones driven by helium shell flashes. It therefore appears important to analyze the possible effect of $\nabla \mu$ in deep-mixing events. To analyze the effect of chemical gradients during deep-mixing events, we performed some simulations of deep-mixing for a model of $0.4641 M_{\odot}$ and $Z=0.02$ with the theory of convection developed by Grossman et al. (1993), in its local version presented by Grossman \& Taam (1996) ${ }^{5}$. This theory incorporates the effect of $\nabla \mu$ both in the determination of the different mixing regimes and the corresponding mixing velocities.

This set of simulations ${ }^{6}$ showed that the inclusion of $\nabla \mu$ prevents the complete penetration of the He-core flash convective zone into the regions of high $\mathrm{H}$-content. In particular, at the $\mathrm{H}$ He transition, the convective zone splits into different convective zones and, consequently, the chemical profile at the $\mathrm{H}-\mathrm{He}$ transition acquires a staircase-like aspect. Eventually some of these convective zones merge and some $\mathrm{H}$ can be burnt. However, the main consequence is that in these simulations almost all the $\mathrm{H}$ rich envelope is not engulfed and burned. In all of these simulations the final abundances remain unchanged after the flash, because even the dilution event is prevented due to the $\mu$-barrier.

However, the theory adopted here is a local theory and does not include any kind of extra-mixing beyond the formal convective boundaries, such as those produced by overshooting (Freytag et al. 1996) or the excitation of gravity waves (Herwig et al. 2006, 2007). Our simulations indicate that the inclusion of an overshooting efficiency as small as $f=0.001$ in our models allows the penetration of the $\mu$-barrier and the development of the H-flash, the surface abundances that arise in these cases are similar to those in which $\mu$ effects are not taken into account. Lower $f$-values are difficult to test in our simulations because they require very high spatial resolution. Although hydrodynamical simulations of convection in homogeneous mediums appears to support the existence of some extra-mixing at convective boundaries, it should be noted that overshooting is expected to be reduced by the $\mu$-barrier itself (Canuto 1998).

\subsection{Effect of diffusion in the outer layers}

We now address the possible effects of the interplay between mass-loss, gravitational settling, chemical diffusion, and radiative levitation in our standard sequences (Tables 2 and 3). It is well known that the abundances of sdB stars depend strongly on the combined effects of diffusion and mass-loss (Unglaub $\&$ Bues 2001), and the same should be true for He-rich subdwarfs (Unglaub 2005). If mass loss is higher than $10^{-12} M_{\odot} \mathrm{yr}$, diffusion would not be able to alter the post-HeCF abundances significantly and the star would remain He-rich during the entire

\footnotetext{
${ }^{5}$ For simulations adopting the convection theory of Grossman et al. (1993), the value of the free parameter $\alpha$ is reduced by ${ }^{4} \sqrt{2}$ from that adopted in the standard MLT due to a difference in the definitions of the relevant coefficients.

${ }^{6}$ We performed several simulations under different numerical assumptions.
}
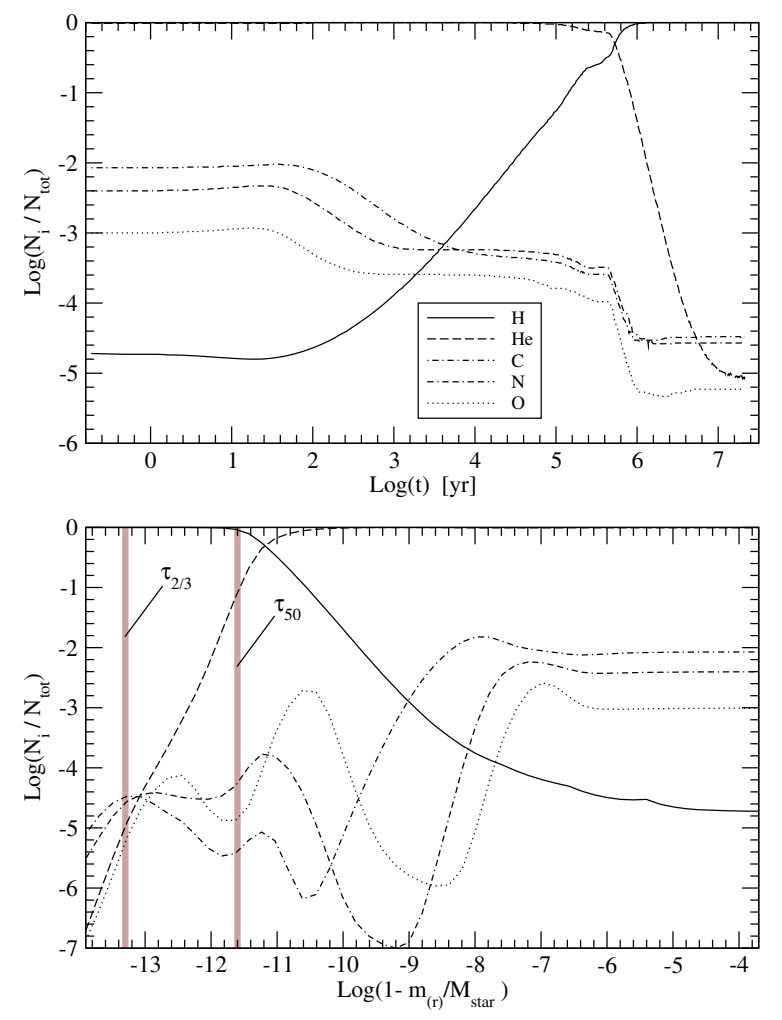

Fig. 7. Top: time evolution of the chemical abundances at $\bar{\tau}=2 / 3$ as a consequence of gravitational settling and radiative levitation. Initial abundances correspond to one of our DM sequences (Table 2; $M=$ $0.48545 M_{\odot}$ and $\left.Z=0.001\right)$ with surface parameters $T_{\text {eff }}=40000 \mathrm{~K}$ and $\log g=6.0$. Bottom: chemical profile of the outer layers after $10^{7} \mathrm{yr}$ when the surface was already H-dominated. Note the very steep chemical profile close to the photosphere $\left(\tau_{2 / 3}\right)$ which can strongly affect the surface values if some mixing process is effective close to the photosphere. In particular note the supersolar He-abundances below $\bar{\tau}=50$ $\left(\tau_{50}\right)$, not far from the location where the bottom of the convective zone due to the He-ionization should be located.

EHB evolution. For the low hydrogen abundances of our DM sequences, a mass-loss rate of approximately $10^{-14} M_{\odot}$ yr would be sufficient to prevent transformation of a He-rich into a H-rich star due to the upward diffusion of hydrogen.

From the theoretical predictions of Vink \& Cassisi (2002) \& Unglaub (2008), it follows that for stars with $M_{*} \approx 0.5 M_{\odot}$, $T_{\text {eff }}=40000 \mathrm{~K}, \log g \lesssim 5.5$, and solar composition, massloss rates of $\dot{M} \gtrsim 10^{-11} M_{\odot}$ yr are expected. The effect of diffusion should therefore be negligible. For more compact stars with $\log g>5.5$, from the theory of radiatively-driven winds, which neglects multi-component effects, weak winds with $\dot{M}<10^{-11} M_{\odot}$ yr are predicted. However, the results of Unglaub (2008) showed that those weak winds cannot be "chemically homogeneous" as assumed in previous investigations. Hydrogen and helium, at least, can hardly be expelled from the star. Therefore, with the method described in Unglaub \& Bues (1998), we performed simulations, in which the absence of any mass-loss was assumed.

In the upper panel of Fig. 7, we show the evolution of the surface abundances (at a Rosseland mean optical depth $\bar{\tau}=2 / 3$ ) 
Table 6. Surface abundances of sequences in which extra-mixing at convective boundaries was considered with different efficiencies $(f)$. The sequences correspond to models with a final mass equal to $0.4641 M_{\odot}$ and $Z=0.02$.

\begin{tabular}{ccccccccc}
\hline \hline$f$-value & $\mathrm{H}$ & $\mathrm{He}$ & ${ }^{12} \mathrm{C}$ & ${ }^{13} \mathrm{C}$ & $\mathrm{N}$ & $\mathrm{O}$ & ${ }^{2} \mathrm{M}_{\mathrm{H}}$ & ${ }^{3} \mathrm{M}_{\mathrm{H}}$ \\
\hline 0.005 & $2.48 \times 10^{-4}$ & 0.9540 & 0.0220 & $4.95 \times 10^{-3}$ & 0.0114 & $5.88 \times 10^{-4}$ & $3.14 \times 10^{-6}$ & $1.93 \times 10^{-6}$ \\
0.01 & $1.18 \times 10^{-8}$ & 0.9548 & 0.0216 & $4.88 \times 10^{-3}$ & 0.0114 & $5.60 \times 10^{-4}$ & $5.05 \times 10^{-10}$ & $9.128 \times 10^{-11}$ \\
0.1 & $1.52 \times 10^{-10}$ & 0.9597 & 0.0227 & $8.41 \times 10^{-5}$ & $7.31 \times 10^{-3}$ & $2.61 \times 10^{-3}$ & $6.85 \times 10^{-12}$ & $1.21 \times 10^{-12}$ \\
\hline
\end{tabular}

predicted by one of our standard DM sequences ${ }^{7}$ (that with $0.48545 M_{\odot}$ and $\left.Z=0.001\right)$ as a consequence of gravitational settling and radiative levitation (at $T_{\text {eff }}=40000 \mathrm{~K}$ and $\log g=6.0$ ). In the absence of mass loss, the star changes from a He-rich subdwarf into a He-deficient one in only $\sim 10^{6} \mathrm{yr}$. Since this timescale is similar to the time it takes the star to reach the ZAHB, surface abundances are expected to become $\mathrm{H}$-dominated as the star settles onto the ZAHB. This leads to the evolutionary channel sketched in Fig. 8., in which He-sdO stars become hot sdB stars due to element diffusion. After about $10^{7} \mathrm{yr}$, the surface abundances are close to the values expected from the equilibrium condition between gravitational settling and radiative levitation obtained by Unglaub \& Bues (1998). This timescale is significantly shorter than the timescales for stellar evolution close to the EHB (65 to $90 \mathrm{Myr}$ ). For higher $\mathrm{H} / \mathrm{He}$ ratios, as predicted by our shallow-mixing sequences, the stellar atmosphere becomes hydrogen-rich on even shorter timescales. For the abundances from the $\mathrm{SM}^{*}$ sequence with $0.47250 M_{\odot}$ and $Z=0.02$, the equilibrium abundances should already be reached by $10^{5} \mathrm{yr}$. In the absence of mass loss or another disturbing processes, the surface composition of the majority of sdB stars should therefore be given by the equilibrium condition between gravitational settling and radiative levitation, independently from the evolutionary scenario from which they originate.

It is well known that the equilibrium abundances of helium are lower than observed (Michaud et al. 1989). The number ratios $\mathrm{He} / \mathrm{H}$ of the hottest $\mathrm{sdB}$ stars are approximately 0.01 (e.g. Lisker et al. 2005). There is therefore a discrepancy of about three orders of magnitude between the predictions and the observations. In Fig. 7, we show the predicted abundances as a function of depth after $10^{7} \mathrm{yr}$. We note the very steep chemical gradient in the outer regions. Within the stellar atmosphere, the helium abundance increases from $\mathrm{He} / \mathrm{H} \approx 10^{-5}$ at $\bar{\tau}=2 / 3$ to supersolar values at $\bar{\tau} \gtrsim 50$ (see Fig. 7). We therefore suggest that the presence of even weak mixing processes (e.g. due to stellar rotation), which tend to smooth concentration gradients, should produce higher helium abundances close to the stellar surface. Thus the large discrepancy for the helium abundance does not necessarily exclude the proposed scenario. In future investigations, it should be shown how mixing processes other than mass loss would affect the upward diffusion of hydrogen, and the gravitational settling of helium in a $\mathrm{H}$-dominated stellar atmosphere. In particular, the possible effect of a thin, outer, convection zone

\footnotetext{
7 With the exception of the $\mathrm{O}$ abundance that was defined to be $\log \left(N_{\mathrm{O}} / N_{\text {tot }}\right)=-3$, instead of $\log \left(N_{\mathrm{O}} / N_{\text {tot }}\right) \sim-5$ as follows from our simulations, for numerical reasons. For an initial value of $\log \left(N_{\mathrm{O}} / N_{\text {tot }}\right) \sim-5$, oxygen moves outward in the outer regions and its abundance tends to its equilibrium value, which is higher than $\log \left(N_{\mathrm{O}} / N_{\text {tot }}\right) \sim-5$. In the underlying regions, however, where oxygen has helium-like configuration, the outward radiative force is negligibly small and oxygen sinks. For an initial abundance of $\log \left(N_{\mathrm{O}} / N_{\text {tot }}\right) \sim-5$, this situation leads to extremely steep concentration gradients in these regions where the flow changes its sign and, as a consequence, to numerical problems.
}

due to changes in ionization of helium, predicted by Groth et al. (1985) at least for supersolar helium abundances, could affect the composition of the stellar atmosphere. For example, in the DM sequence analyzed in this section, stellar evolution models (without diffusion) show that the bottom of this convective region should be located around $\log \left(1-m / M_{\star}\right) \sim-12-$ with the exact location depending on the instantaneous $\log T_{\text {eff }}$ and $\log g$ values - where He abundance is significantly higher (see Fig. 7).

In the $\log T_{\text {eff }}-\log g$ diagram of Fig. 8, the line is shown above which, according to Unglaub (2008), chemical homogeneous winds may exist for stars of solar metallicity. For stars above this line, the mass-loss rates should be sufficiently high to prevent diffusion. The chemical composition should then depend essentially on their evolutionary history. For stars below this "wind limit", no chemically homogeneous winds can exist. The surface composition of these stars should essentially depend on atmospheric processes such as gravitational settling and radiative levitation. Pure metallic winds may possibly still exist, which could produce additional changes in the metal abundances. Due to the complexity of the situation, no reliable quantitative predictions appear to be presently possible. Our results in Fig. 7 indicate, however, that in the absence of mass-loss a helium-rich atmosphere may become a hydrogen-rich one, even for the low hydrogen abundances predicted by the DM sequences.

From Fig. 8, it can be seen that our DM sequences cross the wind limit before the star settles onto the ZAHB. We then expect the onset of diffusion. A similar situation occurs in hot white dwarfs on the upper cooling sequence (Unglaub \& Bues 2000). As soon as winds cease, the abundances of heavy elements decrease and helium-rich white dwarfs became hydrogenrich. We emphasize that the location of the wind limit in the $\log T_{\text {eff }}-\log g$ diagram depends on the chemical composition and the line shown in Fig. 8 is for solar abundances. It would be necessary to investigate the dependence of the mass-loss rates on the abundances of individual elements. From Fig. 8, it can be seen that there is at least qualitative agreement between our predictions and observational results. The helium-poor sdB stars are preferably in the region below the wind limit, where gravitational settling could be effective. The He-rich subdwarfs are preferably above the wind limit, where we expect no diffusion. The composition of these stars should show clear signatures of their evolutionary history.

\section{Discussion}

In Sect. 3.3, we showed that standard sequences lead to final $\mathrm{H}$ abundances (see Table 3) immediately after a DM event that are much lower than inferred for He-sdO stars by Ströer et al. (2007). Only a minority of standard sequences (those which undergo SM/SM* events) display abundances similar to $\mathrm{He}$-sdO stars immediately after the flash. Also, as we have already mentioned, the predicted location in the $g-T_{\text {eff }}$ would differ from that observed. In particular, since the EHB evolution is more than 40 times longer than the evolution from the primary $\mathrm{HeCF}$ to the 


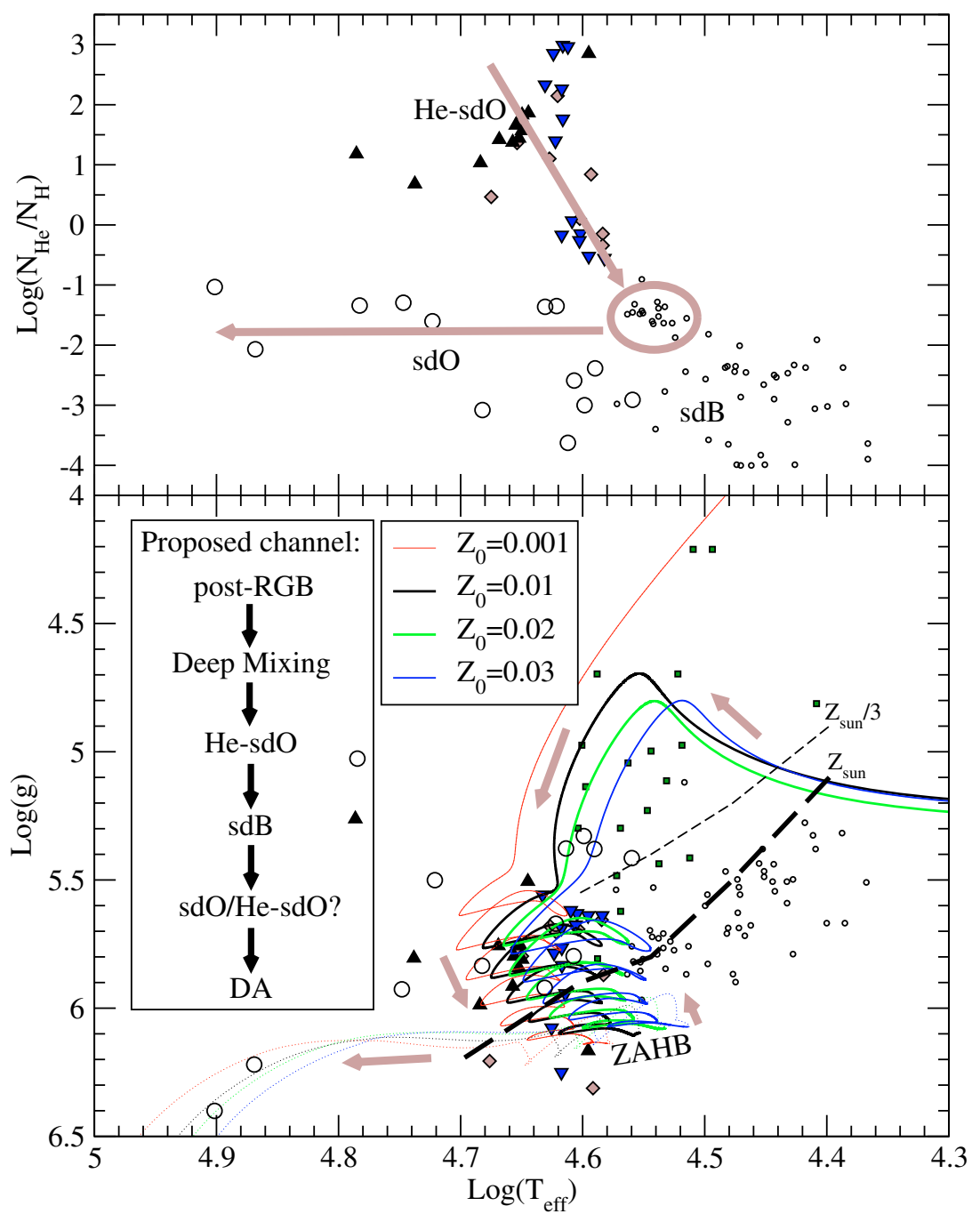

Fig. 8. Sketch of the proposed channel for the evolution after a DM event as a consequence of chemical diffusion in the absence of winds (thick arrows mark the sense of evolution). A similar situation would be expected for post-SM/SM* sequences with weak winds $\left(\dot{M} \sim 10^{-13} M_{\odot} / \mathrm{yr}\right)$. Upper panel: proposed evolution in the abundances- $T_{\text {eff }}$ plane, abundances taken from Ströer et al. (2007) \& Lisker et al. (2005). Lower panel: evolution in the $g-T_{\text {eff }}$ plane. Continuous lines correspond to the evolution as a He-rich subdwarf, while dotted lines mark the evolution after the ZAHB when diffusion would have turned the atmosphere into a $\mathrm{H}$ dominated one. Black dashed lines show the wind limit for solar-like abundances and for two different metallicities (thick line: $Z_{\odot}$, thin line: $Z_{\odot} / 3$ ). (color figure only available in the electronic version)
ZAHB, most of the stars should be clustered around the EHB, which is not observed. However, there are some viable scenarios in which our results are consistent with observations. These scenarios depend on the existence or not of weak homogeneous winds in He-rich subdwarf stars as we now discuss.

As mentioned in Sect. 4.4, in a very recent study Unglaub (2008) showed that weak homogeneous winds are impossible in hot subdwarfs at high gravities. In this case, as shown in Fig. 7, the low amount of $\mathrm{H}$ remaining in our standard sequences after a DM event is sufficient to convert a He-rich to a He-deficient surface composition in about $10^{6} \mathrm{yr}$. Within this picture, after the DM event the star first evolves towards the ZAHB star as a Herich subdwarf. Then, when gravity has increased sufficiently, the star crosses the wind limit causing homogeneous winds to halt, then diffusion leads to an increase in the $\mathrm{H}$ abundance as the star evolves towards the ZAHB (both processes occur on timescales of the order of $1 \mathrm{Myr}$ ). We note in particular (Fig. 7) that in only a few thousand years the $\mathrm{H}$ abundance rises from its post-DM value to the values usually measured in He-sdO stars (Ströer et al. 2007) and thus the correct $\mathrm{H}$-abundances are predicted. When the HB has been reached the evolutionary timescale becomes more than a factor of 40 longer (65 to $90 \mathrm{Myr}$, see Table 2) and the star turns into a $\mathrm{H}$-dominated sdB star. This possible evolutionary channel is sketched in Fig. 8. In this case no clustering of He-sdO stars should be expected close to the EHB, in good agreement with the results of Ströer et al. (2007). It is interesting to note that the effective temperatures expected for these stars, when on the HB (and with a H-dominated atmosphere), agree with those of the hotter sdB stars (which also have higher $\mathrm{He}$ abundances than their cooler counterparts). The conversion of surface abundances with $C>N$ into surfaces with $N>C$ as $\mathrm{H}$ diffuses outwards, might also be related (see Figs. 7 and 8) to the lack of C-rich He-sdO stars with lower $N_{\mathrm{He}} / N_{\mathrm{H}}$ values in the study of Ströer et al. (2007). Within this scenario and in the absence of any other channel that creates hot-subdwarfs, the number ratio of He-sdO stars to hot $\left(T_{\text {eff }} \gtrsim 33500\right) \mathrm{sdB}$ stars should be of the order of $1 / 40$ in a complete sample (although higher ratios can still be possible if some process that disturbs diffusion is present). Once the star finishes the He-core burning stage (or EHB), it moves above the wind limit again (see Fig. 8) and homogeneous winds again become possible. If this is so, since the H-rich layer is very thin, a new conversion into a He-sdO star might happen (now at higher surface gravities and temperatures). Finally, it is interesting to note in Fig. 7 that the $\mathrm{C}$-abundance decrease rapidly by about a factor of 25 and the star changes its surface composition from $C>N$ to $C<N$. This occurs while the star is still strongly $\mathrm{H}$-deficient. We note that, at this point (between $t=1000$ and $10000 \mathrm{yr}$ in Fig. 7), the star displays surface abundances qualitatively similar $\left(H \sim 2.5 \times 10^{-3}\right.$, $N \sim 2 \times 10^{-3}$ and $C<N$ by mass fraction), although quantitatively different, to those inferred by Lanz et al. (2004), for the peculiar star LB 1766.

In the case of post-SM/SM* stars our simulation indicates that the atmosphere should turn into a H-dominated one in about 
$1000 \mathrm{yr}$ from the moment in which homogeneous winds cease. Then, if homogeneous winds do not exist at high gravities, post$\mathrm{SM} / \mathrm{SM}^{*}$ stars would only be He-rich while they are above the wind limit (see Fig. 8). This is clearly not the case for most HesdO stars in the sample of Ströer et al. (2007) but it does describe the observed surface properties of JL87 $-T_{\text {eff }}=29000 \mathrm{~K}$ and $\log g=5.5$, Lanz et al. (2004); $T_{\text {eff }}=26000 \mathrm{~K}$ and $\log g=4.8$, Ahmad et al. (2007).

We emphasize that the possible conversion of He-sdO stars into hot sdB stars in the HB discussed in the previous paragraphs is interesting, not only because it predicts the correct distribution in the $\log g-T_{\text {eff }}$ diagram but also in view of the significantly higher He abundances of sdB stars with $T_{\text {eff }} \gtrsim 33500 \mathrm{~K}$ compared with their cooler counterparts, which might reflect differences in their previous evolution.

In contrast, if the effects of element diffusion were of minor importance for all He-rich subdwarfs, then only the abundances resulting from $\mathrm{SM} / \mathrm{SM}^{*}$ events would be consistent with the surface properties measured for He-sdO stars (Ströer et al. 2007), unless DM abundances differ strongly from those predicted by the standard MLT theory (see Sect. 4.1). We note that the fraction of stars undergoing SM/SM* events corresponds to only a small fraction of hot-flashers in standard sequences. In this context, our results in Sect. 4.3 indicate that the role of chemical gradients should be analyzed to determine whether the fraction of shallow-mixing episodes might be increased by the effect of chemical gradients that tend to prevent the penetration of convection into the H-rich envelope.

The hot-flasher scenario therefore can qualitatively predict the correct surface properties and distribution of He-sdO stars. It is then interesting to note that the lack of close binaries (with periods of the order of days as observed for sdB stars) in He-sdO stars is also expected naturally within the hot-flasher scenario. Very close binaries with final periods of the order of days will indeed fill their Roche lobes before reaching the RGB-tip and never undergo the HeCF. Also, it is not clear if it would be possible to have hot-flashers within a common envelope system. In any case, in order to have a hot-flasher within a close binary system, a very fine tuning of the initial masses and periods seems to be needed. Consequently, post hot-flasher H-deficient stars should not be usual in these systems. The lack of close binary systems with He-sdO components should then not be taken as an argument against the hot-flasher and in favor of the merger scenario. In contrast, wide systems would be a much better way of distinguishing between both possible evolutionary origins for $\mathrm{He}$-sdO stars, because they should be more probable within the hot-flasher picture.

\section{Final remarks}

We have performed 1D stellar evolution simulations of the hotflasher scenario for a wide variety of cases and metallicities. Based on these results, we have given a detailed description of the surface characteristics of standard sequences. For these sequences, we have studied how abundances could be altered by element diffusion processes. We have then extended the scope of our work by studying deviations from standard assumptions. Our results can be summarized as follows:

- We have carried out an ample exploration of the parameter space of the hot-flasher scenario that will allow far more effective tests of its predictions. Our results confirm the partial results by Cassisi et al. (2003); Lanz et al. (2004), for the deep-mixing and shallow-mixing events respectively.
- Our deep-mixing sequences have abundances similar to those recently inferred for PG1544+488, confirming that this star can be the result of a DM event. Our results also confirm the result of Lanz et al. (2004) that JL87 abundances are compatible with a recent SM event for this star. None of our sequences show abundances similar to those inferred for LB 1766 and this object is therefore probably not the result of a recent hot-flasher event. However, it is worth noting that after a DM event, element diffusion might produce abundances qualitatively similar to those of LB 1766.

- Standard hot-flasher sequences predict $\mathrm{C} / \mathrm{N}$ ratios in the range from $\sim 9.5$ to $\sim 0.85$. This is particularly interesting in the light of the $\mathrm{N}$-rich and $\mathrm{C}$-rich classes described by Ströer et al. (2007), although no clear correlation is found between those classes and our sequences in the $g-T_{\text {eff }}$ diagram. In the absence of weak winds, however, the lack of C-rich He-sdO stars with lower $\mathrm{He} / \mathrm{H}$ values in the sample of Ströer et al. (2007) can be qualitatively understood in terms of element diffusion.

- The location of our sequences in the $g-T_{\text {eff }}$ diagram show qualitative agreement with the parameters inferred by Ströer et al. (2007) for He-sdO, while they predict (as already noted by Ströer et al. 2007) that these stars should cluster around the EHB, which is not observed. An interesting (and plausible, in view of the results of Unglaub 2008) possibility for this discrepancy to be removed is if $\mathrm{He}$-sdO stars evolve from a region in the $g-T_{\text {eff }}$ diagram where homogeneous winds can exist, into a region where such winds are impossible. This should lead to the onset of H-diffusion which, as we have showed in Sect. 4.4, allows the conversion of He-sdO stars close to the ZAHB into H-dominated sdB stars even for the low abundances resulting from DM events. This scenario is extensively discussed in Sect. 5.

In the presence of weak homogeneous winds only the surface properties arising from $\mathrm{SM} / \mathrm{SM}^{*}$ episodes would be consistent with those inferred for He-sdO stars, unless DM abundances differ strongly from those predicted by the standard MLT with diffusive convective mixing.

- In all cases in which a conversion of He-sdO into hot $\mathrm{H}$ rich sdB stars is possible, we have shown that the hot-flasher scenario reproduces correctly the observed properties (abundances and their distribution in the $T_{\text {eff }}-g$ plane) of He-sdO stars and links them, as immediate progenitors, to the hotter sdB stars. This is interesting in view of the higher Heabundances of the hotter sdB stars.

- We have argued that the lack of close binaries among He-sdO stars cannot be used as an argument against the hot-flasher scenario because this dearth is also naturally expected within this scenario.

- Our study of departures from standard assumptions shows that the role of chemical gradients as an extra barrier to the penetration of the inner convective zone into the H-rich envelope has to be studied in detail. This is particularly important in determining if the fraction of SM/SM* episodes is higher than predicted by standard sequences. In contrast, extra-mixing processes alone does not seem to produce significant departures from standard predictions.

We conclude that the hot-flasher scenario is a viable explanation of the formation of He-rich subdwarfs and that the understanding of some key points needs to be clarified before a final statement can be made. Special attention should be paid to analyzing the existence and intensity of disturbing processes, such as winds and turbulence, in the outer layers of He-rich subdwarfs. Also, 
the search for wide binaries among He-sdO stars is necessary to distinguish between the predictions of the merger and hotflasher scenarios. Finally, a better understanding of the burning and mixing of $\mathrm{H}$ in the hot interior (probably by means of hydrodynamical simulations of the event) is badly needed.

Acknowledgements. Part of this work was supported by PIP 6521 grant from CONICET. M3B thanks professor U. Heber for instructive correspondence, and professor O. Benvenuto and Dr. A. De Vito for useful comments on binary evolution. Also the Max-Planck Institut für Astrophysik in Garching and the European Association for Research in Astronomy are gratefully acknowledged for an EARA-EST fellowship under which this work was started. Finally, we want to thank our referee, S. Cassisi, for his comments and suggestions which have improved the quality and readability of the article.

Note added in proof. After acceptance, the authors became aware of the close binary nature of PG 1544+488 (Ahmad, A. \& Jeffery, C. S. 2007, ASPC, 391, 261). This fact raises serious doubts on a possible deep-mixing origin for this star.

\section{References}

Ahmad, A., Behara, N., Jeffery, C., Sahin, T., \& Woolf, V. 2007, A\&A, 465, 541 Alexander, D., \& Ferguson, J. 1994, ApJ, 437, 879

Althaus, L. G., Serenelli, A. M., Córsico, A. H., \& Montgomery 2003, A\&A, 404, 593

Althaus, L. G., Serenelli, A. M., Panei, J. A., et al. 2005, A\&A, 435, 631

Angulo, C., Arnould, M., Rayet, M., et al. 1999, Nucl. Phys. A, 656, 3

Brown, T., Sweigart, A., Lanz, T., Landsman, W., \& Hubeny, I. 2001, ApJ, 562, 368

Casagrande, L., Flynn, C., Portinari, L., Girardi, L., \& Jimenez, R. 2007, MNRAS, 382, 1516

Calamida, A., Corsi, C., Bono, G., et al. 2008, ApJ, 673, L29

Canuto, V. 1998, ApJ, 508, 103

Castellani, M., \& Castellani, V. 1993, A\&A, 407, 649

Castellani, M., Castellani, V., \& Prada Moroni, P. 2006, A\&A, 457, 569

Cassisi, S., Schlattl, H., Salaris, M., \& Weiss, A. 2003, ApJ, 582, L43

Cassisi, S., Potekhin, A., Pietrinferni, A., Catelan, M., \& Salaris, M. 2007, ApJ, 661, 1094

D’Cruz, N., Dorman, B., Rood, R., \& O’Connell, R. 1996, ApJ, 466, 359

D'Cruz, N., O'Connell, R., Rood, R., Whitney, H., \& Dorman, B. 2000, ApJ, 530,352

de Boer, K., Drilling, J., Jeffery, C. S., \& Sion, E. M. 1997, Third Conference on Faint Blue Stars, L. Davis Press, 515
Espey, B., \& Crowley, C. 2008, ASPC, in press [arXiv: 0803. 1170] Flynn, C. 2004, Publication of the Astron. Soc. of Australia, 21, 126 Freytag, B., Ludwig, H.-G., \& Steffen, M. 1996, A\&A, 313, 497 Grevesse, N., \& Sauval, A. J. 1998, Space Sci. Rev., 85, 161 Grossman, S., \& Taam, R. 1996, MNRAS, 283, 1165

Grossman, S., Narayan, R., \& Arnett, D. 1993, ApJ, 407, 284 Groth, H. G., Kudritzky, R. P., \& Heber, U. 1985, A\&A 152, 107 Haft, M., Raffelt, G., \& Weiss, A. 1994, ApJ, 425, 222

Herwig, F., Blöcker, T., Schönberner, D., \& El Eid, M. 1997, A\&A, 324, L81 Herwig, F. 2000, A\&A, 360, 952

Herwig, F., Freytag, B., Fuchs, T., et al. 2007, ASPC, 378, 43

Herwig, F., Freytag, B., Hueckstaedt, R., \& Timmes, F. 2006, ApJ, 642, 1057

Hirsch, H., Heber, H., \& O'Toole, S. 2008, ASP Conf. Ser., 392, 175

Iglesias, C., \& Rogers, F. 1996, ApJ, 464, 943

Iben, I. 1984, ApJ, 277, 333

Iben, I., \& Mac Donald, J. 1995, Lect. Notes Phys., 443 (Berlin: SpringerVerlag), 48

Jeffery, C. S. 2008, ASP Conf. Ser., 391, 3

Lanz, T., Brown, T., Sweigart, A., Hubeny, I., \& Landsman, W. 2004, ApJ, 602, 342

Lemke, M., Heber, U., Napiwotzki, R., Dreizler, S., \& Engels, D. 1997, Third Conference on Faint Blue Stars (L. Davis Press), 375

Lisker, T., Heber, U., Napiwotzki, R., et al. 2005, A\&A, 430, 223

Michaud, G., Bergeron, P., Heber, U., \& Wesemael, F. 1989, ApJ, 338, 417

Miller Bertolami, M. M., \& Althaus, L. G. 2007, MNRAS, 380, 763

Miller Bertolami, M. M., Althaus, L. G., Serenelli, A. M., \& Panei, J. A. 2006, A\&A, 449, 313

Moehler, S., Sweigart, A., Landsman, W., \& Dreizler, S. 2002, A\&A, 395, 37

Moehler, S., Sweigart, A., Landsman, W., Hammer, N., \& Dreizler, S. 2004, A\&A, 415, 313

Moehler, S., Dreizler, S., Lanz, T., et al. 2007, A\&A, 475, L5

Saio, H., \& Jeffery, S. 2000, MNRAS, 313, 671

Salaris, M., \& Weiss, A. 2002, A\&A, 388, 492

Schröder, K.-P., \& Cuntz, M. 2005, ApJ, 630, L73

Serenelli, A., \& Fukugita, M. 2007, ApJS, 172, 649

Ströer, A., Heber, U., Lisker, T., Napiwotzki, R., \& Dreizler, S. 2005, ASP Conf. Ser., 334, 309

Ströer, A., Heber, U., Lisker, T., et al. 2007, A\&A, 462, 269

Sweigart, A. 1997, Third Conference on Faint Blue Stars (L. Davis Press), 3

Unglaub, K. 2005, ASP Conf. Ser., 334, 297

Unglaub, K. 2008, A\&A, 486, 923

Unglaub, K., \& Bues, I. 1998, A\&A, 338, 75

Unglaub, K., \& Bues, I. 2000, A\&A, 359, 1042

Unglaub, K., \& Bues, I. 2001, A\&A, 374, 570

Vink, J., \& Cassisi, S. 2002, A\&A, 392, 553

Woodward, P., Herwig, F., Porter, D., Fuchs, T., et al. 2008, AIPC, 990, 300

Yi, Sukyoung, \& Yoon, Suk-Jin 2004, Ap\&SS, 291, 205 